\title{
Maternal dietary resveratrol alleviates weaning-associated intestinal inflammation and diarrhea in porcine offspring by altering intestinal gene expression and microbiota
}

\author{
Qingwei Meng ${ }^{1}$, Shishuai Sun ${ }^{1}$, Shiqi He ${ }^{1}$, Baoming Shi ${ }^{1}$, Anshan Shan ${ }^{1}$ and Baojing \\ Cheng ${ }^{1}$ \\ ${ }^{1}$ Institute of Animal Nutrition, Northeast Agricultural University, Harbin 150030, P.R. China
}

Correspondence to: Anshan Shan, email: asshan@neau.edu.cn

Keywords: resveratrol; inflammation; microbiota; piglets; diarrhea

Received: October 27, $2017 \quad$ Accepted: January 02, $2018 \quad$ Published: January 02, 2018

Copyright: Meng et al. This is an open-access article distributed under the terms of the Creative Commons Attribution License 3.0 (CC BY 3.0), which permits unrestricted use, distribution, and reproduction in any medium, provided the original author and source are credited.

\section{ABSTRACT}

Early weaning commonly results in gastrointestinal disorders, inflammation and diarrhea in infants and young animals. Resveratrol, a plant phenol, affords protection against inflammation and cancer. A porcine model was used to investigate the effects of maternal resveratrol supplementation on diarrhea, intestinal inflammation and intestinal morphology in offspring during weaning. Fecal microbiota and intestinal gene expression were measured by $16 \mathrm{~S}$ rDNA sequencing and RNA sequencing (RNAseq) analysis, respectively. Maternal resveratrol supplementation alleviated weaningassociated intestinal inflammation and diarrhea, and improved intestinal morphology in the offspring. In weaning piglets, maternal resveratrol supplementation increased the proportions of butyrate-producing bacteria, such as Flavonifractor, Odoribacter and Oscillibacter, as determined by 16S rDNA sequencing. RNA-seq analysis identified 189 and 139 differentially expressed genes (DEGs) in weaning and post-weaning piglets, respectively. Kyoto Encyclopedia of Genes and Genomes (KEGG) pathway analysis showed that DEGs were enriched for the $T$ cell receptor, primary immunodeficiency, mitogen-activated protein kinase (MAPK) and Ras signaling pathways in weaning piglets and for the cytokine-cytokine receptor interaction pathway and metabolismrelated pathways in post-weaning piglets.

\section{INTRODUCTION}

Weaning is a critical period in young mammals because of an increased susceptibility to gastrointestinal disorders, inflammation and diarrhea. The post-weaning period is characterized by an immediate, transient drop in food intake resulting in severe undernutrition and growth inhibition, which consequentially inhibits various aspects of small intestinal architecture and function and can lead to gut-associated disorders and diarrhea $[1,2]$. Antibiotics have been effectively used to treat diarrhea in children and animal neonates after weaning over the past several decades [3]. However, excessive antibiotics use has accelerated the emergence of multidrug-resistant microbes, which has been shown to damage the health of humans and animals. Thus, alternatives to antibiotics and additional measures to reduce the current use of antibiotics are urgently needed. Dietary substances, such as prebiotics, sodium butyrate and plant extracts, have been shown to control weaning-associated diarrhea and improve intestinal health in humans and animals. Traditional measures of alleviating weaning-associated intestinal dysfunction have focused on dietary manipulation during pre- and/or post-weaning stages. However, recent studies have indicated that maternal dietary supplementation can improve the immune status and gastrointestinal health of offspring. Zhong et al. [4] reported that the maternal consumption of methionine promoted neonatal intestinal growth by increasing morphological development and upregulating the expression of genes responsible for nutrient 
metabolism. In addition, maternal dietary seaweed extract and fish oil are beneficial for the intestinal microflora, intestinal morphology and immune status of weaned pigs [5]. Thus, beneficial maternal dietary treatments may potentially reduce or alleviate weaning-associated intestinal dysfunction and diarrhea.

Resveratrol, a component of grapes, berries, peanuts and herbal medicines, is a polyphenol belonging to the stilbene family of phytoalexins, which are antibiotic compounds produced by plants in response to infection $[6,7]$. Resveratrol has generated intense scientific and public interest in recent years, mainly because of its significant beneficial effects in the treatment of cancer, type 2 diabetes, cardiovascular disease and aging $[6,8-10]$. In addition, resveratrol has been shown to alter the intestinal barrier [11] and intestinal morphology [12]. Liu et al. [13] reported that dietary supplementation with resveratrol improved villus morphology, increased the numbers of goblet cells and lymphocytes, and attenuated the mRNA expression of heat shock proteins and nuclear transcription factor $\mathrm{NF}-\kappa \mathrm{B}$ in the jejunum of chickens exposed to heat stress. Fidalgo et al. [14] demonstrated that dietary supplementation with resveratrol attenuated chronic colonic inflammation, reduced PGES-1, COX-2 and iNOS expression and down-regulated the p-38 MAPK pathway in colonic mucosa in a dextran sulfate sodium-induced colitis mouse model. In recent years, maternal resveratrol has been used as a therapeutic agent for pregnancy complications in rodent models of preeclampsia [15], fetal growth restriction [16] and gestational diabetes [17]. In addition, maternal supplementation with resveratrol has been shown to alleviate oxidative stress and apoptosis in diabetic dam offspring and reduce oxidative stress, inflammation and metabolic dysfunction of offspring in a maternal protein restriction model $[18,19]$. However, to our knowledge, there are no available data assessing the impact of maternal resveratrol supplementation on the intestinal health and inflammation status of the offspring.

Gastrointestinal microbiota has a profound influence on host health through regulation of intestinal nutritional metabolism, maturation of the immune system, and establishment of the gut barrier [20]. Beginning shortly after birth, microbiota communities that assemble in this ecosystem have been shown to be remarkably hostspecific and broadly stable over time [21]. In most cases, the bacterial composition of intestinal content is stable and protects host from pathogens; however, this composition can be influenced by many factors, particularly dietary factors. The weaning process for pigs involves an abrupt dietary shift from sow milk to a completely feed-based diet that produces abrupt taxonomic and functional shifts in the gut microbiota [22]. Resveratrol, a phytoalexin, has been shown to influence the gut microbiota in several intestinal disease models [23, 24]. However, the impact of maternal dietary resveratrol supplementation on the gut microbiota in offspring remains unknown.
The pig (porcine) has been proposed as the best non-primate model for human nutrition based on its omnivorous nature and similarities in size and gut physiology [25]. In the present study, we explored the effects of maternal dietary resveratrol supplementation during pregnancy and lactation on the intestinal inflammation and diarrhea status of offspring during weaning in a pig model. Furthermore, we performed RNA-seq analysis to measure intestinal gene expression and $16 \mathrm{~S}$ rDNA gene sequencing to evaluate microbiota composition in feces.

\section{RESULTS}

\section{Fecal score and growth performance}

The fecal score was characterized using a 5-point fecal consistency scoring system: (1) hard, dry pellet; (2) firmed, formed stool; (3) soft, most stool that retain its shape; (4) soft, unformed stool; and (5) watery liquid that can be poured. Liquid consistency (a score of 4-5) was considered indicative of diarrhea [26]. During days 15-21 after birth, the fecal scores of piglets were reduced by maternal dietary resveratrol supplementation $(P<0.05$, Figure 1A). During the days 3-5 post-weaning, diarrhea was observed (score $>4$ ); however, the diarrhea was alleviated by maternal resveratrol supplementation.

In our study, the litter size (control (Con): 11.32 \pm 0.60 vs. resveratrol (Res): $11.94 \pm 0.54$ ) and birth weight (Con: $1.48 \pm 0.06$ vs. Res: $1.47 \pm 0.07$ ) were not significantly different between the two treatments. However, the average weight daily gains of piglets during days 14-21 and 21-28 were increased by maternal resveratrol supplementation (Figure 1B), indicating that maternal resveratrol is beneficial for the growth performance of piglets.

\section{Intestinal morphology}

The intestinal morphology of jejunum is shown in (Figure 2A, 2D-2F). The villus height and villus/crypt ratio in the jejunum were reduced and crypt depth was increased by weaning $(P<0.01)$, which was consistent with a previous study [27]. The villus height and villus/crypt ratio of the jejunum in weaning and postweaning piglets were increased by maternal resveratrol supplementation $(P=0.01)$.

The weaning and post-weaning piglets from the Con treatment showed shorter intestinal villi compared to piglets from Res treatment (Figure 2B). In addition, the post-weaning piglets showed damaged and atrophic villi, which were improved by maternal resveratrol supplementation. In addition, the microvillus height in weaning and post-weaning piglets was increased by maternal dietary resveratrol (Figure 2C, 2G). These results 
A

$$
\begin{aligned}
& \rightarrow \text { Con } \\
& \mp \text { Res }
\end{aligned}
$$

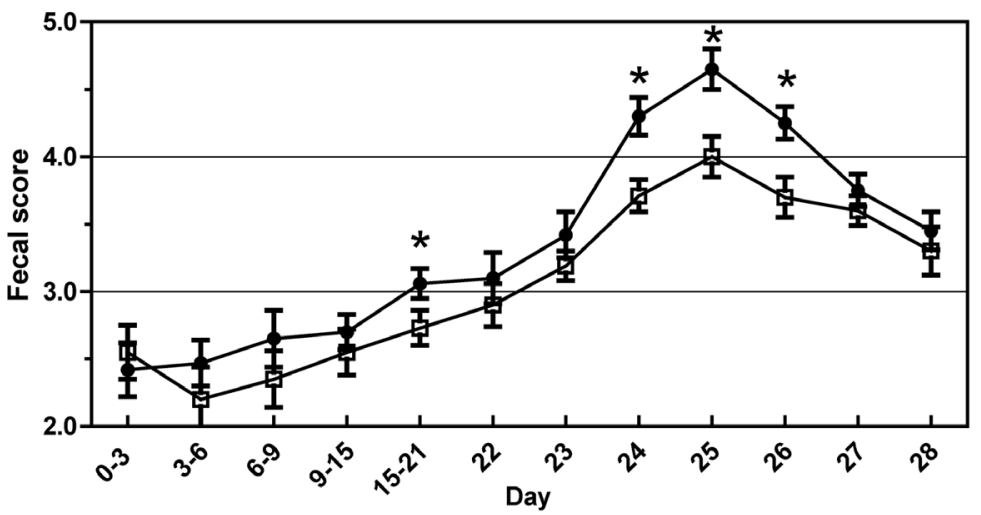

B

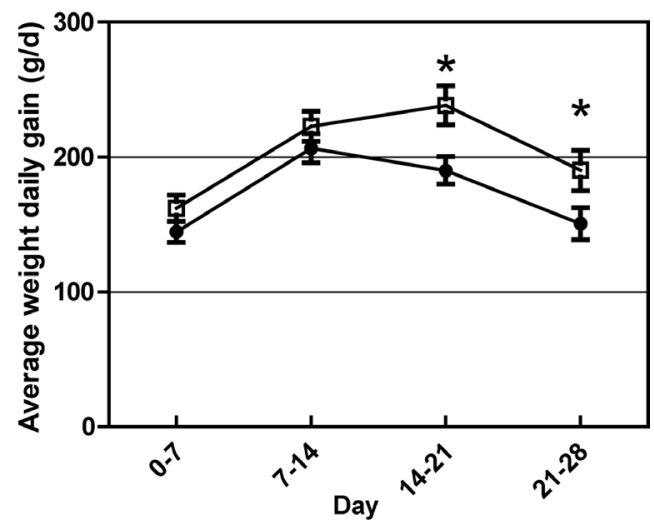

Figure 1: Fecal score and average daily weight gains of piglets. (A) Fecal score. (B) Average daily weight gains. Con, control treatment; Res, resveratrol treatment. Piglets were weaned at 21 days after birth. All values are expressed as the mean $\pm \operatorname{SEM}(n=20) .{ }^{*} P<0.05$.

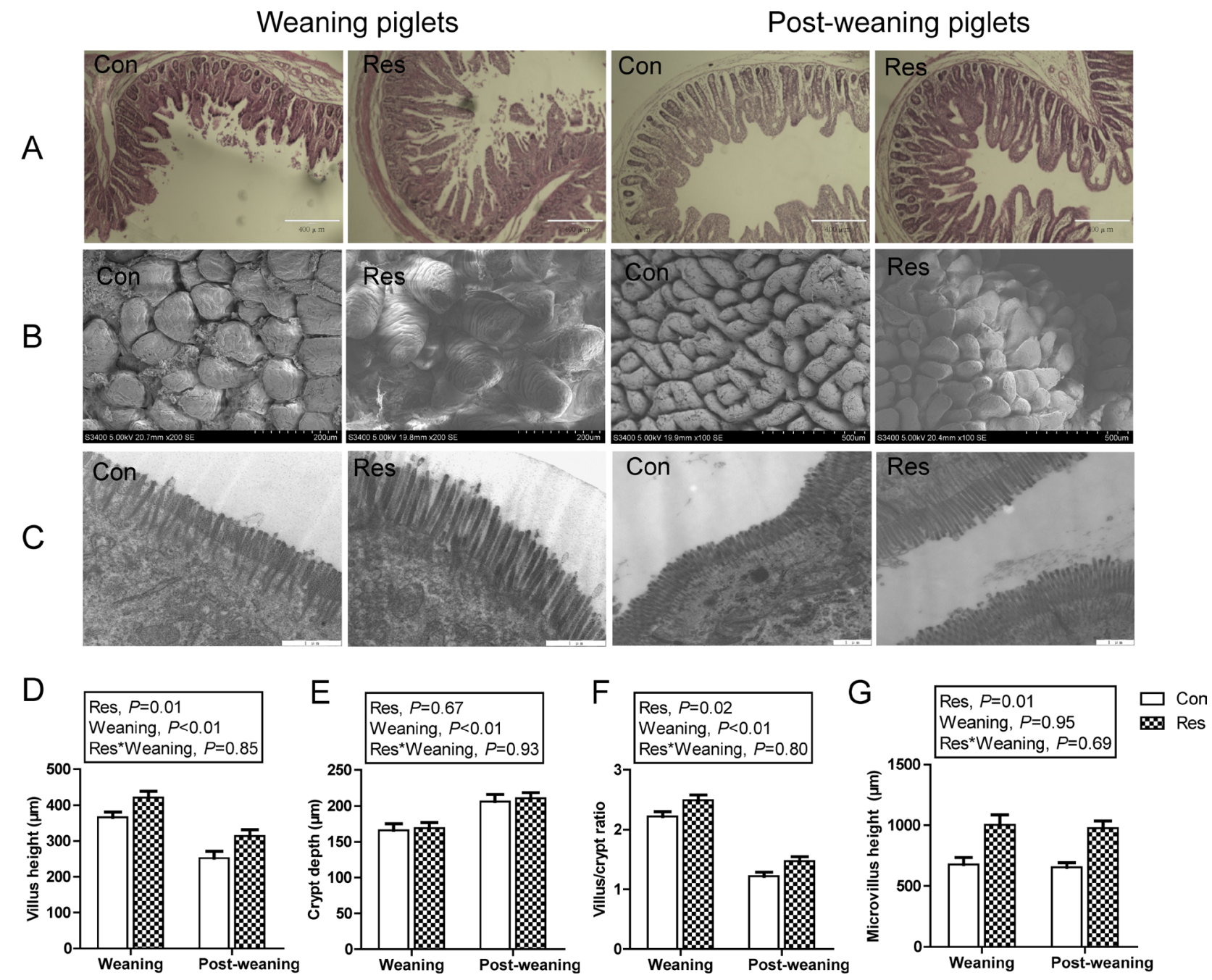

Figure 2: Intestinal morphology of weaning piglets and post-weaning piglets. (A) H\&E staining. (B) Scanning electron micrographs. (C) Transmission electron microscopy. (D) Villus height. (E) Crypt depth. (F) Villus/crypt ratio (G) Microvillus height. Con, control treatment; Res, resveratrol treatment; Res*Weaning, interaction between resveratrol and weaning. All values are expressed as the mean $\pm \mathrm{SEM}$. 
Table 1: Effects of maternal dietary resveratrol supplementation on the intestinal pro-inflammatory cytokines in weaning and post-weaning piglets ${ }^{1,2}$

\begin{tabular}{|c|c|c|c|c|c|c|c|c|}
\hline \multirow{2}{*}{ Item } & \multicolumn{2}{|c|}{ Weaning } & \multicolumn{2}{|c|}{ Post-weaning } & \multirow{2}{*}{ SEM } & \multicolumn{3}{|c|}{$P$-value } \\
\hline & Con & Res & Con & Res & & Res & Weaning & Res*Weaning \\
\hline IL-1 $\beta$ (pg/mg prot) & $2.09^{\mathrm{ab}}$ & $2.14^{\mathrm{a}}$ & $2.17^{\mathrm{a}}$ & $1.63^{b}$ & 0.07 & 0.64 & 0.11 & 0.03 \\
\hline IL-6 (pg/mg prot) & 9.06 & 4.90 & 13.39 & 7.43 & 0.85 & $<0.01$ & 0.01 & 0.47 \\
\hline IL-8 (pg/mg prot) & 2.58 & 2.58 & 2.48 & 2.45 & 0.03 & 0.87 & 0.06 & 0.82 \\
\hline TNF $-\alpha$ (pg/mg prot) & 5.69 & 5.45 & 5.99 & 4.73 & 0.17 & 0.02 & 0.48 & 0.09 \\
\hline
\end{tabular}

${ }^{1}$ All of the values are expressed as the means and pooled SEM, $n=6$; , b Means without a common letter differ $(P<0.05)$.

${ }^{2}$ Con, control treatment; Res, resveratrol treatment; Res*Weaning, interaction between resveratrol and weaning; IL-1 $\beta$, interleukin $1 \beta$; IL-6, interleukin 6; IL-8, interleukin 8; TNF- $\alpha$, tumor necrosis factor $\alpha$.

suggest that maternal resveratrol improves the intestinal morphology in weaning and post-weaning piglets.

\section{Intestinal pro-inflammatory cytokines}

As shown in Table 1, we found that interleukin-6 (IL-6) levels were significantly increased $(P=0.01)$ by weaning, indicating that weaning induced inflammation in intestine, which is in agreement with a previous study [28]. In addition, we observed that the IL-1 $\beta$ levels in post-weaning piglets were reduced by maternal resveratrol supplementation (weaning * resveratrol, $P=0.03$ ). IL-6 and tumor necrosis factor $\alpha$ TNF- $\alpha$ levels in both weaning and post-weaning piglets were decreased by $(P<0.05)$ maternal resveratrol supplementation. These data indicate that maternal resveratrol supplementation has beneficial effects on intestinal inflammation of piglets during the weaning period.

\section{Summary of microbiota analysis}

The intestinal microbiota exerts profound influences on the intestinal immune system and health status. We found that the diarrhea and inflammation in piglets during weaning process were alleviated by maternal resveratrol supplementation. To further understand these effects, we performed $16 \mathrm{~S}$ rDNA gene sequencing to measure microbiota in the feces of weaning and postweaning piglets. The weaning and post-weaning piglets from each treatment were named W-con, W-Res, PWCon and PW-Res, with a total of 22 samples with 5-6 biological replicates in each group. Across all samples, 833,405 quality sequences were of sufficient quality for analysis. On average, 37,882 sequences per sample with a read length greater than $300 \mathrm{bp}$ were obtained (Supplementary Table 1). The top 10 phyla and genera in terms of relative abundance of the fecal bacteria are shown in Figure 3. In weaning piglets, Bacteroidetes and Firmicutes were the most prevalent phyla in both Con and Res treatments, followed by Proteobacteria and Fusobacteria (Figure 3A); the most prevalent genera were Prevotella and Fusobacterium, followed by Alloprevotella and Bacteroides (Figure 3B). In postweaning piglets, the most prevalent phyla were also Bacteroidetes and Firmicutes, followed by Proteobacteria and Actinobacteria (Figure 3C). At the genus level, Prevotella and Lactobacillus were most prevalent. The second-highest genera were unclassified Prevotellaceae and unclassified Ruminococcaceae (Figure 3D).

\section{Differences in microbiota composition}

Alpha diversity measurements of the fecal microbiota community, including the Shannon, Simpson, and Chaol indices, were not affected by maternal resveratrol supplementation or weaning (Supplementary Table 2). At the phylum level, we failed to observe any influence of maternal resveratrol treatment on the microbiota composition of weaning and post-weaned piglets (Figure 4). There were no interactions between resveratrol and weaning for microbiota composition at the phylum level. However, the microbiota composition was partially modulated by weaning. The abundance of Firmicutes was significantly increased $(P=0.05)$ by weaning, and post-weaning piglets showed a higher abundance of Firmicutes than weaning piglets. In addition, the abundances of Proteobacteria, Cyanobacteria, Verrucomicrobial and Lentisphaerae were significantly reduced $(P<0.05)$ by weaning, and post-weaning piglets had lower abundances of these phyla than weaning piglets. The abundance of Fusobacteria tended to be reduced by weaning $(P=0.06)$.

At the genus level (Figure 5), the abundance of Flavonifractor was increased $(P<0.05)$ by maternal resveratrol treatment. The abundance of Escherichia also tended to be increased by maternal resveratrol treatment. There were interactions between resveratrol supplementation and weaning for the abundance of Oscillibacter, Odoribacter and Lachnobacterium (resveratrol * weaning, $P<0.05$ ). In weaning piglets, 
the abundances of Oscillibacter, Odoribacter and Lachnobacterium were increased by maternal resveratrol treatment but were decreased by maternal resveratrol treatment in post-weaning piglets. The abundances of Faecalibacterium, Blautia, Clostridium sensu stricto, Ruminococcus, Clostridium XIVa, Roseburia, Butyricicoccus, CF231 belonging to the Prevotellaceae family, Coprococcus, Clostridium XIVb, Lachnospiracea incertae, Clostridium IV, Paraprevotella and Peptococcus were increased $(P<0.05)$ by weaning. The abundances of Fusobacterium, Bacteroides, Campylobacter, Sutterella,
Escherichia, Megasphaera, Anaerobiospirillum, Butyricimonas and Oligosphaera were decreased $(P<$ 0.05 ) by weaning.

\section{Summary of RNA-seq}

To further detect differences in the intestinal functions of weaning and post-weaning piglets between Con and Res treatment, we performed RNA-seq analysis using the Illumina sequencing platform. The weaning and post-weaning pigs from each treatment group were
A

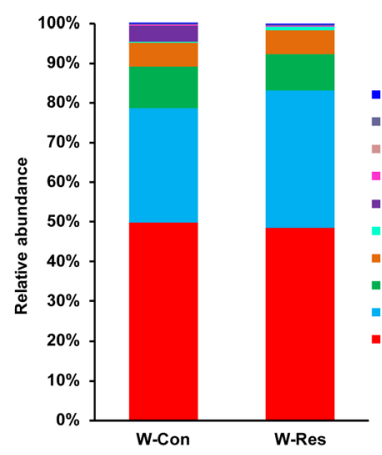

C

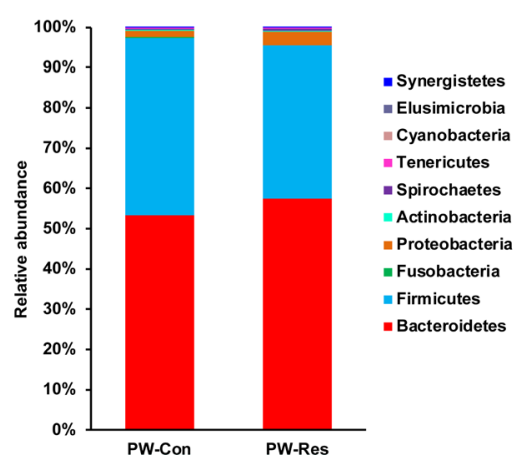

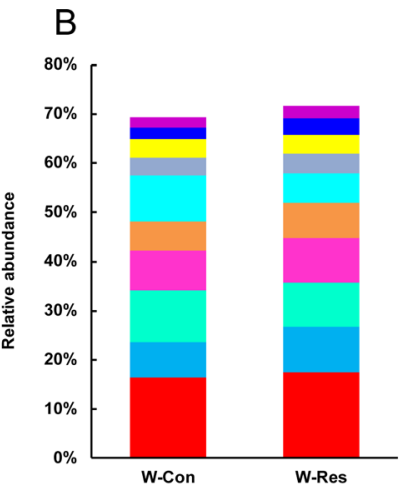

- p_Bacteroidetes;c_Bacteroidia;o_Bacteroidales;f_Prevotellaceae;g_unclassified - p_Bacteroidetes;c__Bacteroidia;o_Bacteroidales;f_Porphyromonadaceae;g_unclassified

p_Firmicutes;C_Clostridia;o_Clostridiales;f_unclassified;g_unclassified «p_Bacteroidetes;c__Bacteroidia;o__Bacteroidales;f_unclassified; g_unclassified " $\mathbf{p} \_$_Bacteroidetes;c__Bacteroidia;o__Bacteroidales;f_Prevotellaceae;g_Alloprevotella -p_Firmicutes;c_Bacilli;o_Lactobacillales;f_Lactobacillaceae;g_Lactobacillus - $\mathbf{p} \_$B_acteroidetes;c_Bacteroidia;o_Bacteroidales; $f$ _Bacteroidaceae; $g \_B a c t e r o i d e s$ " $\mathbf{p} \_$Fusobacteria;c_Fusobacteriia;o_Fusobacteriales;f_Fusobacteriaceae;g_Fusobacterium -p_Firmicutes;c_Clostridia;o_Clostridiales;f_Ruminococcaceae;g_unclassified - p_Bacteroidetes;c_Bacteroidia;o_Bacteroidales;f_Prevotellaceae;g_Prevotella
D

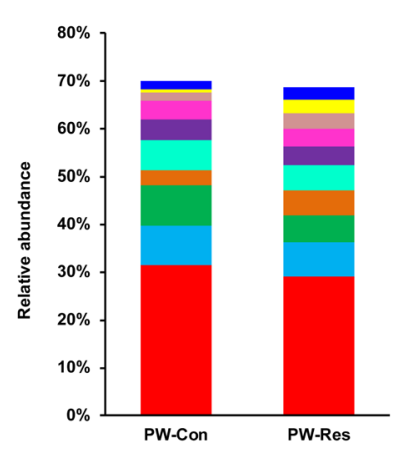

- $\mathrm{p}$ _BBacteroidetes;c_Bacteroidia;o__Bacteroidales;f_Porphyromonadaceae;g_Barnesiella P_Bacteroidetes;c_Bacteroidia;o__Bacteroidales;f_Bacteroidaceae;g_Bacteroides mp_Bacteroidetes;C_Bacteroidia;O__Bacteroidales;f_Porphyromonadaceae; = $\mathrm{P} \_$_Bacteroidetes;c_Bacteroidia;0__Bacteroidales; $;$_unclassified; $\mathbf{g} \_$unclassified - P_Firmicutes;c_Clostridia;o_Clostridiales;___Lachnospiraceae;g_unclassified n p_Firmicutes;c_Clostridia;o_Clostridiales;f_Ruminococcaceae;g_unclassified = P_Bacteroidetes;c_Bacteroidia;o__Bacteroidales;f_Prevotellaceae;g_Alloprevotella $\sim \mathrm{p}$ _Firmicutes;c__Bacilli;o_Lactobacillales;f_Lactobacillaceae;g_Lactobacillus = P_Bacteroidetes;c_Bacteroidia;0__Bacteroidales; $f$ _Prevotellaceae;g_unclassified I P_Bacteroidetes;c_Bacteroidia;o__Bacteroidales;f_Prevotellaceae;g_Prevotella

Figure 3: Fecal microbiota composition of weaning piglets and post-weaning piglets. (A) The top 10 phyla of fecal bacteria in weaning piglets. (B) The top 10 genera of fecal bacteria in weaning piglets. (C) The top 10 phyla of fecal bacteria in post-weaning piglets. (D) The top 10 genera of fecal bacteria in post-weaning piglets. Con, control treatment; Res, resveratrol treatment; W-Con, weaning piglets from control treatment; W-Res, weaning piglets from resveratrol treatment; PW-Con, post-weaned piglets from control treatment; PW-Con, post-weaned piglets from resveratrol treatment.

A

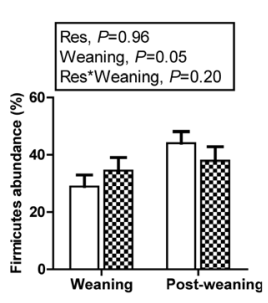

B

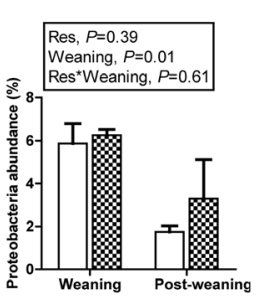

C

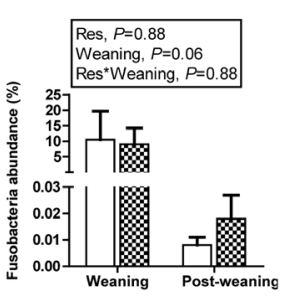

D

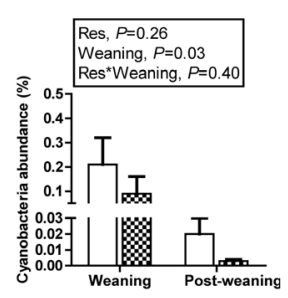

$E$

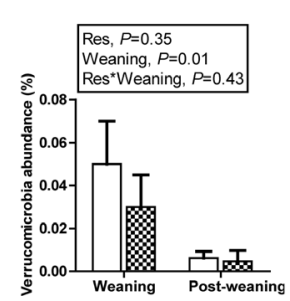

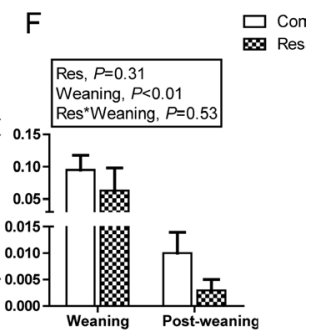

Figure 4: Fecal microbiota affected by maternal dietary resveratrol and/or weaning at the phylum level. (A) Firmicutes. (B) Proteobacteria. (C) Fusobacteria. (D) Cyanobacteria. (E) Verrucomicrobia. (F) Lentisphaerae. Con, control treatment; Res, resveratrol treatment; Res*Weaning, interaction between maternal resveratrol and weaning. All values are expressed as the mean $\pm \mathrm{SEM}(n=5-6)$. 
named W-Con, W-Res, PW-Con and PW-Res, with a total of 12 samples with three biological replicates in each group. The main characteristics of the 12 libraries are summarized in Supplementary Table 3. After removing adaptor, ambiguous and low-quality sequences, an average of 48,732,086 valid clean data reads remained. These 12 libraries contained 49,418,207 raw reads on average. The average valid data ratio is $98.60 \%$ and $77.77 \%$ of the valid reads mapped to the database (Supplementary Table 4), including $48.91 \%$ unique mapped reads and $28.86 \%$ multimapped reads.

\section{Differential gene expression between dietary treatments}

To identify differentially expressed genes (DEGs), we used $\mid \log 2$ (fold-change) $\mid>1$ and $P$-value $<0.05$ as standards. Volcano plots (Figure 6A, 6B) were generated to visualize the distribution of expressed genes between dietary treatments. The red dots in the volcano plot represent up-regulated genes, and blue dots represent down-regulated genes. There were 70 up-regulated genes and 119 down-regulated genes between the W-Con and

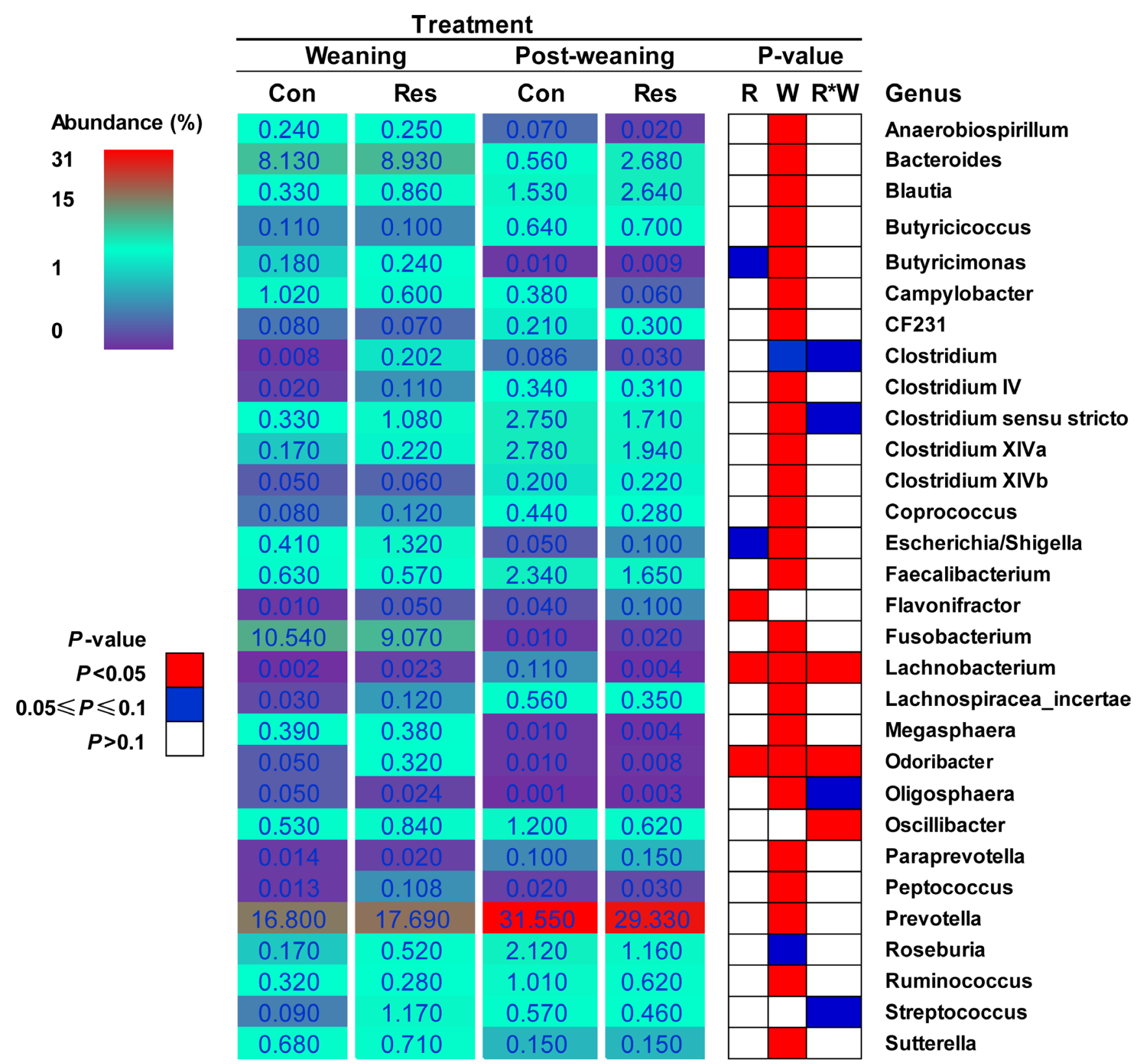

Figure 5: Fecal microbiota affected by maternal dietary resveratrol and/or weaning at the genus level. All of the values are expressed as the mean $\pm \operatorname{SEM}(n=5-6)$. Con, control treatment; Res, resveratrol treatment; $\mathrm{R}$, main effect of resveratrol; W, main effect of weaning; $\mathrm{R}^{*} \mathrm{~W}$, interaction between resveratrol and weaning. 
W-Res treatments (Res/Con, Supplementary Table 5). In addition, there were 72 up-regulated genes and 67 down-regulated genes between the PW-Con and PWRes treatments (Res/Con, Supplementary Table 6). The distributions of DEGs in weaning piglets (Figure 6C) and post-weaning piglets (Figure 6D) were depicted in heat maps.

To validate the results of RNA-seq analysis, qRTPCR was employed to determine the relative expression of 8 genes in weaning piglets (Figure 7A), including CCL5, DHX-58, RHOH, PGLYRP2, IL-10, F2, THBS1 and LTF; and 8 genes in post-weaning piglets (Figure 7B), including CCR7, CYBRD1, IL-10RA, RDH5, NOS2, GZMA, EMP2, OLFML3. GAPDH was used as internal control gene, as it did not respond to dietary treatments. In weaning piglets, CCL5, RHOH, IL-10, and THBS1 were significantly down-regulated; while DHX-58, F2, and LTF were up-regulated by maternal resveratrol $(P$ $<0.05$ ), which was consistent with the data of RNA-seq analysis. PGLYRP2 was not significantly affected by maternal resveratrol, but showed a similar trend. In postweaning piglets, CCR7, IL-10RA and NOS2 were down-

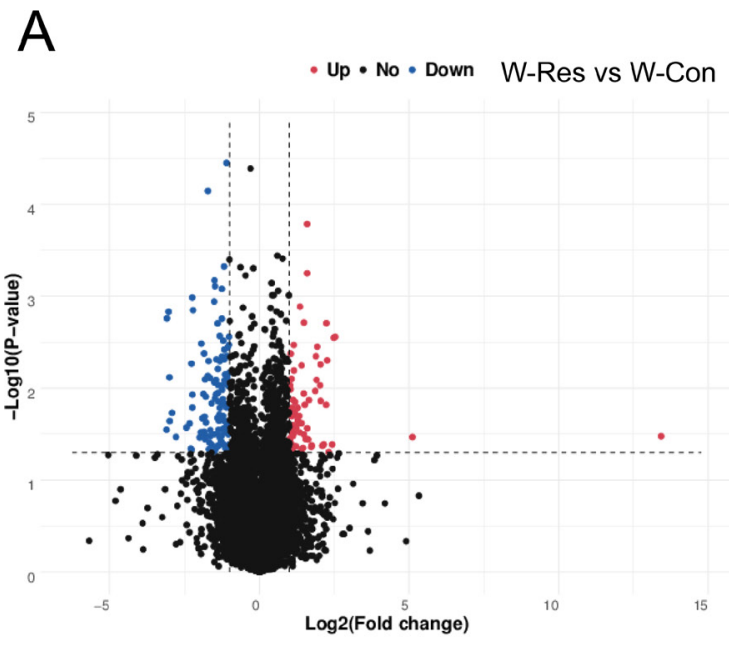

B
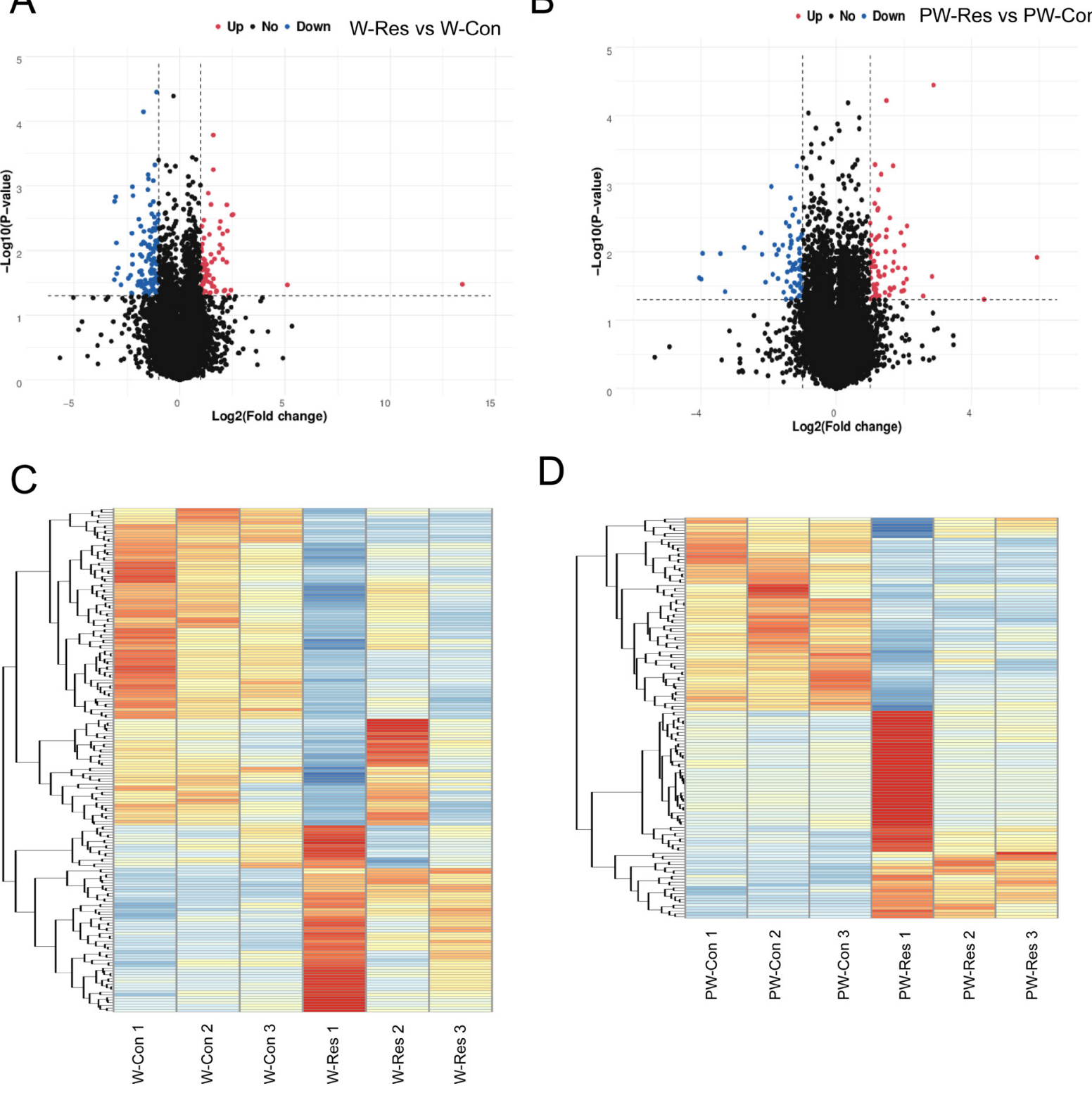

$\mathrm{D}$

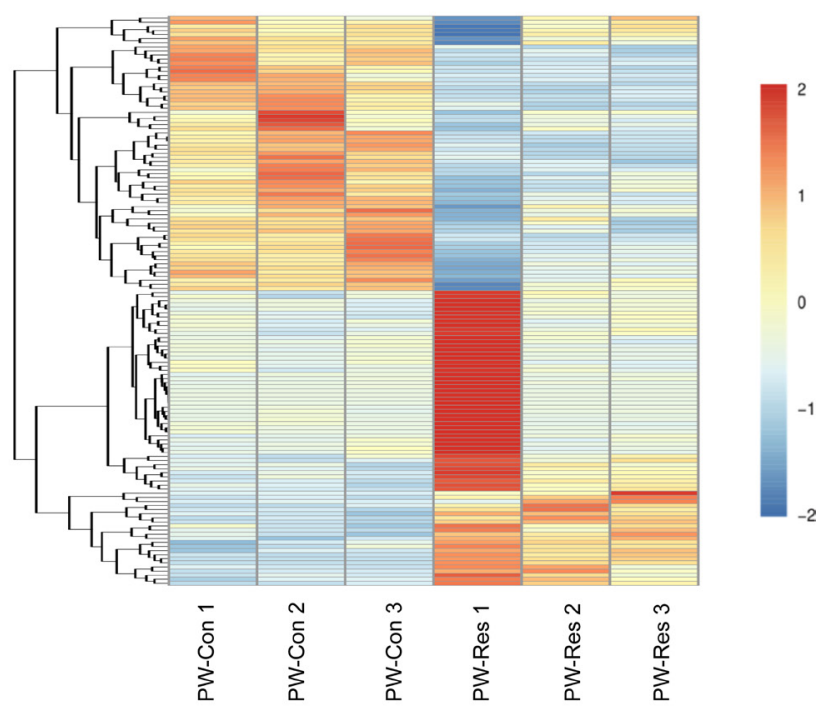

Figure 6: Volcano plots and hierarchical clustering heat-maps of DEGs between Con and Res treatments. (A) Volcano plot of DEGs in weaning piglets. (B) Volcano plot of DEGs in post-weaning piglets. (C) Hierarchical clustering heat map of DEGs in weaning piglets. (D) Hierarchical clustering heat map of DEGs in post-weaning piglets. The fold-changes were calculated as Res (mean of three biological replicates)/Con (mean of three biological replicates). Con, control treatment; Res, resveratrol treatment; W-Con, weaning piglets from control treatment; W-Res, weaning piglets from resveratrol treatment; PW-Con, post-weaned piglets from control treatment; PW-Con, post-weaned piglets from resveratrol treatment. 
regulated; while CYBRD1, RDH5 and OLFML3 were upregulated by maternal resveratrol $(P<0.05)$, which was in accordance with the data of RNA-seq analysis. GZMA was not significantly influenced by maternal resveratrol but showed a similar trend. EMP2 showed a similar trend compared with RNA-seq analysis and tended to be upregulated by maternal resveratrol $(P=0.07)$. Differences in magnitude of fold-change values were likely due to differences in detection sensitivity of the two methods [29]. In addition, correlation analysis demonstrated that the values of $\log 2$ (fold-change) obtained from RNAseq and qRT-PCR were significantly correlated in both weaning piglets (Pearson $\mathrm{R}^{2}=0.93, P<0.001$ ) and postweaning piglets (Pearson $\mathrm{R}^{2}=0.94, P<0.001$ ). Thus, our RNA-seq analysis results are valid.

\section{Gene ontology (GO) enrichment analysis of DEGs}

To gain insight into the biological processes that could be differentially regulated with different maternal diets, we performed GO enrichment analysis with 189 DEGs in weaning piglets and 139 DEGs in post-weaning piglets.

In weaning piglets, 231 biological process terms, 54 molecular function terms, and 26 cellular component terms we significantly enriched $(P<0.05$, Supplementary Table 7). The top 10 GO terms (based on the number of genes) are shown in Figure 8A. Terms related to GTPase activity, GTPase signal transductions, and intracellular signal transduction, were predominant in the biological
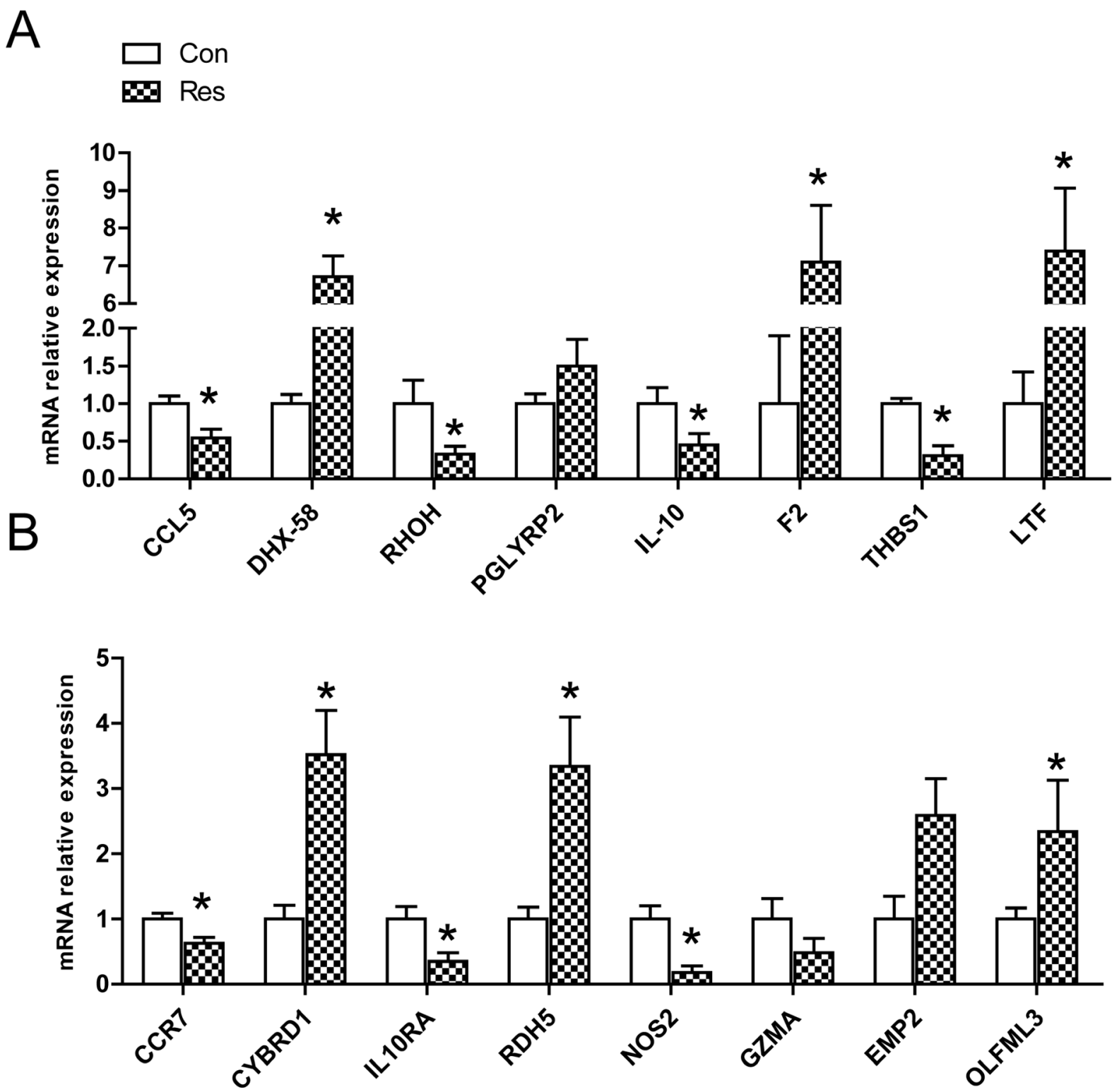

Figure 7: Validation of RNA-seq results with qRT-PCR analysis. (A) Selected genes in weaning piglets. (B) Selected genes in post-weaning piglets. The mRNA levels of selected genes were analyzed by qRT-PCR and normalized to GAPDH. All values are expressed as the mean $\pm \operatorname{SEM}(n=3) .{ }^{*} P<0.05$. Con, control treatment; Res, resveratrol treatment. 
process, followed by the immune response, inflammatory response, and cell migration, adhesion and differentiation. Regarding the classification of molecular function, several DEGs were involved in magnesium ion binding, actin binding and actin filament binding. Terms regarding the cytoplasm predominantly included in cellular component, followed by intracellular and external sides of the plasma membrane. In addition, several terms involved in immunity and intestinal structure, such as the actin cytoskeleton, cell-cell junction, alpha-beta $\mathrm{T}$ cell receptor complex and immunological synapse, were enriched in weaning piglets.

In post-weaning piglets, 160 biological process terms, 24 cellular component terms and 48 molecular function terms were significantly enriched $(P<0.05)$ (Supplementary Table 8). The top 10 GO terms (based on the number of genes) are shown in Figure 8B. In postweaning piglets, GO analysis showed the terms including oxidation-reduction process, inflammatory response, chemokine-mediated signaling pathway, response to lipopolysaccharide, regulation of apoptotic process and apoptotic signaling pathway which belong to biological process, were enriched. The term oxidoreductase activity was predominant in the molecular function classification. In the classification of cellular component, the term of cell surface was predominant followed by the external side of plasma membrane and the nucleosome.

\section{KEGG pathway analysis of DEGs}

In weaning piglets, by matching DEGs to the KEGG database, we identified 11 pathways $(P<0.05$, Table 2$)$, including the $\mathrm{T}$ cell receptor, primary immunodeficiency,

A

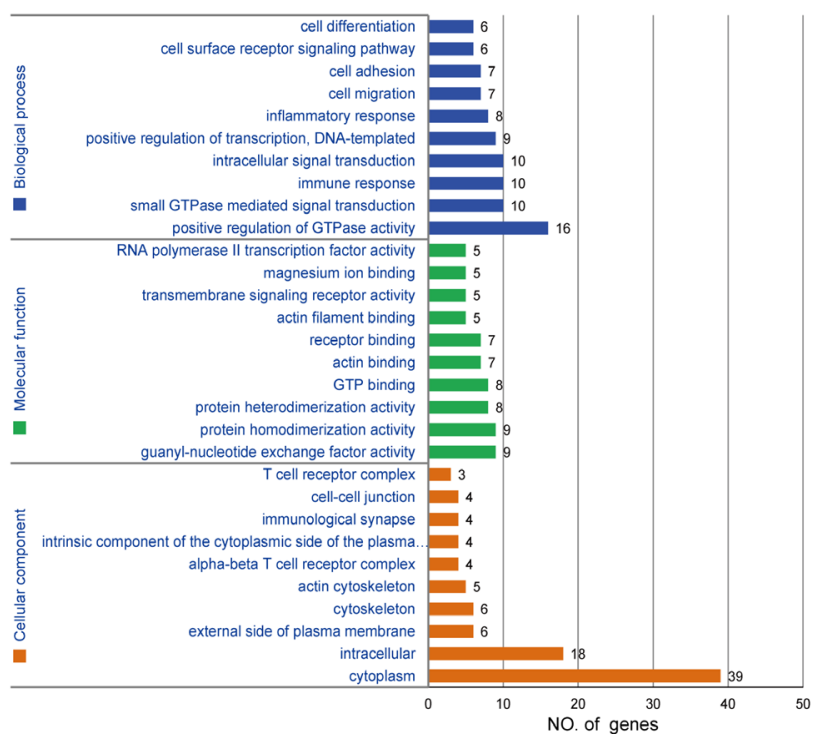

intestinal immune network for IgA production, hematopoietic cell lineage, MAPK, Ras, regulation of actin cytoskeleton and cell adhesion molecules pathways. In addition, three human disease pathways were enriched, including Chagas disease, systemic lupus erythematosus and amoebiasis.

In post-weaning piglets, 9 pathways were significantly $(P<0.05)$ enriched (Table 3$)$. These pathways were mostly related to nutrition metabolism and absorption, such as retinol metabolism, arachidonic acid metabolism, vitamin digestion and absorption, glyoxylate and dicarboxylate metabolism, and glycine, serine and threonine metabolism. In addition, the cytokine-cytokine receptor interaction pathway and 3 human disease pathways were enriched.

\section{Interaction networks between proteins encoded by DEGs}

The known and predicted interaction networks between proteins encoded by DEGs were identified using the STRING database. The interaction network used proteins as its nodes, and assigned an edge between two proteins if they interacted with one another. These interactions contained direct (physical) and indirect (functional) interactions based on numerous sources, such as experimental repositories or computational prediction methods. In weaning piglets, there were 39 hubs that were found to interact with more than one protein (Figure 9). As shown, a dense region of protein interaction was observed with FGR (interacted with 18 proteins, RHOBTB2 (interacted with 16 proteins), $\mathrm{RHOH}$ (interacted with 16 proteins), and PRKCQ (interacted with

B

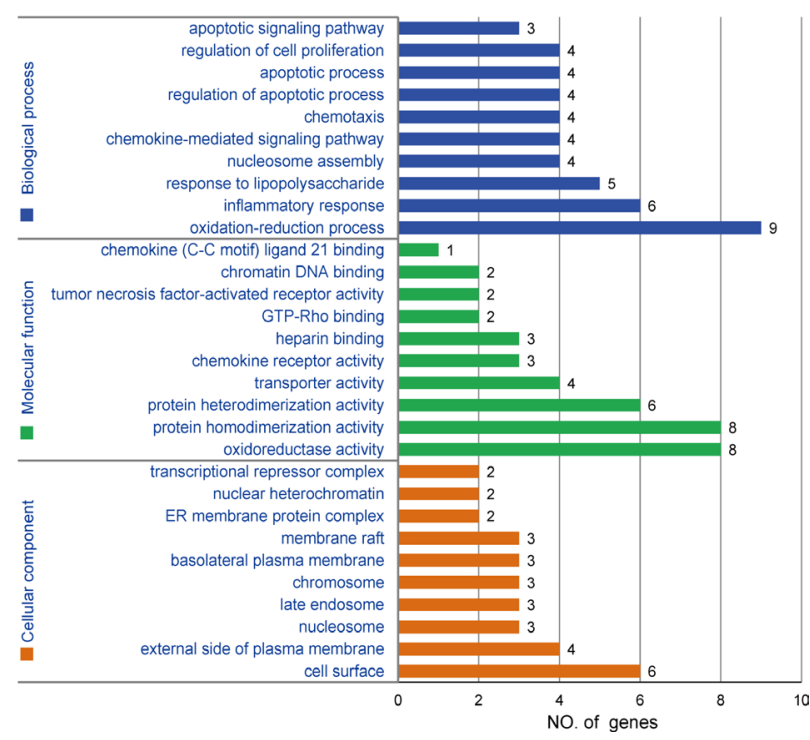

Figure 8: GO functional enrichment analysis of DEGs between Con and Res treatment. (A) GO functional enrichment analysis of DEGs in weaning piglets. (B) GO functional enrichment analysis of DEGs in post-weaning piglets. The top 10 terms in each classification are shown; for the complete dataset, please see Supplementary Tables 7 and 8. 
Table 2: KEGG pathway enrichment of DEGs in weaning piglets ${ }^{1}$

\begin{tabular}{|c|c|c|c|c|}
\hline Pathway ID & Pathway name & Down-regulated genes & Up-regulated genes & $P$-value \\
\hline ko04660 & $\begin{array}{l}\text { T cell receptor signaling } \\
\text { pathway }\end{array}$ & $\begin{array}{c}\text { CD247/CD3D/CD3E/CD3G/ICOS/ } \\
\text { IL10/LCP2/ } \\
\text { RASGRP1/ENSSSCG00000006851/ } \\
\text { ENSSSCG00000010908 }\end{array}$ & - & $<0.01$ \\
\hline ko05340 & $\begin{array}{c}\text { Primary } \\
\text { immunodeficiency }\end{array}$ & $\begin{array}{c}\text { CD3D/CD3E/ENSSSCG00000010908/ } \\
\text { ICOS }\end{array}$ & - & $<0.01$ \\
\hline ko05142 & Chagas disease & $\begin{array}{c}\text { CCL5/CD247/CD3D/CD3E/CD3G/ } \\
\text { IL10 }\end{array}$ & - & 0.00 \\
\hline ko04672 & $\begin{array}{l}\text { Intestinal immune } \\
\text { network for IgA } \\
\text { production }\end{array}$ & ICOS/IL10/ITGB7/SLA-DRB1 & - & 0.00 \\
\hline ko04514 & $\begin{array}{l}\text { Cell adhesion molecules } \\
\text { (CAMs) }\end{array}$ & $\begin{array}{l}\text { CD2/ENSSSCG00000010908/ICOS/ } \\
\text { ITGB/SELL/SLA-DRB1 }\end{array}$ & - & 0.00 \\
\hline ko05322 & $\begin{array}{l}\text { Systemic lupus } \\
\text { erythematosus }\end{array}$ & $\begin{array}{c}\text { ENSSSCG00000024003/H3F3A/IL10/ } \\
\text { SLA-DRB1 }\end{array}$ & - & 0.01 \\
\hline ko04010 & $\begin{array}{l}\text { MAPK signaling } \\
\text { pathway }\end{array}$ & $\begin{array}{l}\text { MEF2C/NF1/PPM1A/RASA2/ } \\
\text { RASGRF1/RASGRP1 }\end{array}$ & HSPB1 & 0.01 \\
\hline ko04640 & $\begin{array}{l}\text { Hematopoietic cell } \\
\text { lineage }\end{array}$ & $\mathrm{CD} 2 / \mathrm{CD} 3 \mathrm{D} / \mathrm{CD} 3 \mathrm{E} / \mathrm{CD} 3 \mathrm{G}$ & - & 0.02 \\
\hline ko04014 & Ras signaling pathway & $\begin{array}{l}\text { ETS1/NF1/RASA2/RASAL3/ } \\
\text { RASGRF1/RASGRP1 }\end{array}$ & - & 0.02 \\
\hline ko04810 & $\begin{array}{l}\text { Regulation of actin } \\
\text { cytoskeleton }\end{array}$ & $\begin{array}{c}\text { CFL2/ENSSSCG00000006851/ITGAE/ } \\
\text { ITGB7/PIP4K2A }\end{array}$ & $\mathrm{F} 2$ & 0.02 \\
\hline ko05146 & Amoebiasis & ARG2/ENSSSCG00000024003/IL10 & HSPB1 & 0.04 \\
\hline
\end{tabular}

${ }^{1}$ DEGs, differentially expressed genes between control treatment and resveratrol treatment.

Table 3: KEGG pathway enrichment of DEGs in Post-weaning piglets ${ }^{1}$

\begin{tabular}{|c|c|c|c|c|}
\hline Pathway ID & Pathway name & Down-regulated genes & Up-regulated genes & $P$-value \\
\hline ko00830 & Retinol metabolism & ENSSSCG00000016303 & LRAT/RDH5/SDR16C5 & 0.00 \\
\hline ko05152 & Tuberculosis & $\begin{array}{c}\text { CALM1/CD209/ } \\
\text { ENSSSCG00000000884/NOS2/ } \\
\text { FCER1G/IL10RA, }\end{array}$ & - & 0.00 \\
\hline ko05133 & Pertussis & C4A/CALM1/IRF1/NOS2 & - & 0.00 \\
\hline ko00590 & Arachidonic acid metabolism & PLA2G2D & $\begin{array}{c}\text { ENSSSCG00000013842/ } \\
\text { GGT5 }\end{array}$ & 0.01 \\
\hline ko04977 & $\begin{array}{l}\text { Vitamin digestion and } \\
\text { absorption }\end{array}$ & - & LRAT/SLC5A6 & 0.01 \\
\hline ko04060 & $\begin{array}{c}\text { Cytokine-cytokine receptor } \\
\text { interaction }\end{array}$ & $\begin{array}{c}\text { CCR7/CXCL13/CXCR3/ } \\
\text { CXCR4/ } \\
\text { IL10RA/TNFRSF4 }\end{array}$ & - & 0.02 \\
\hline ko00630 & $\begin{array}{l}\text { Glyoxylate and dicarboxylate } \\
\text { metabolism }\end{array}$ & - & GLYCTK/HYI & 0.02 \\
\hline ko05222 & Small cell lung cancer & ENSSSCG00000000884/NOS2 & RXRG & 0.02 \\
\hline ko00260 & $\begin{array}{l}\text { Glycine, serine and threonine } \\
\text { metabolism }\end{array}$ & - & $\begin{array}{l}\text { ENSSSCG00000020981/ } \\
\text { GLYCTK }\end{array}$ & 0.04 \\
\hline
\end{tabular}

${ }^{1}$ DEGs, differentially expressed genes between control treatment and resveratrol treatment. 
15 proteins). The interacting proteins involved in immune functions included LCP2, CD3D, CD2, CD247, CD3E and CD3G. The cytokine IL-10 interacted with ARG2, CCL5, CSF2RB, F2, PTPRC and SELL.

In post-weaning piglets, there were 27 hubs that were found to interact with more than one protein
(Figure 10). The protein-protein interaction network in post-weaning piglets demonstrated that TOPOII and $\mathrm{H} 2 \mathrm{AFZ}$ interacted with nine and six proteins, respectively. In this network, interactions were observed between $\mathrm{G}$ protein-coupled receptors and chemokine receptors including S1PR2, CCR7, CXCL13, CXCR3, and CXCR4.

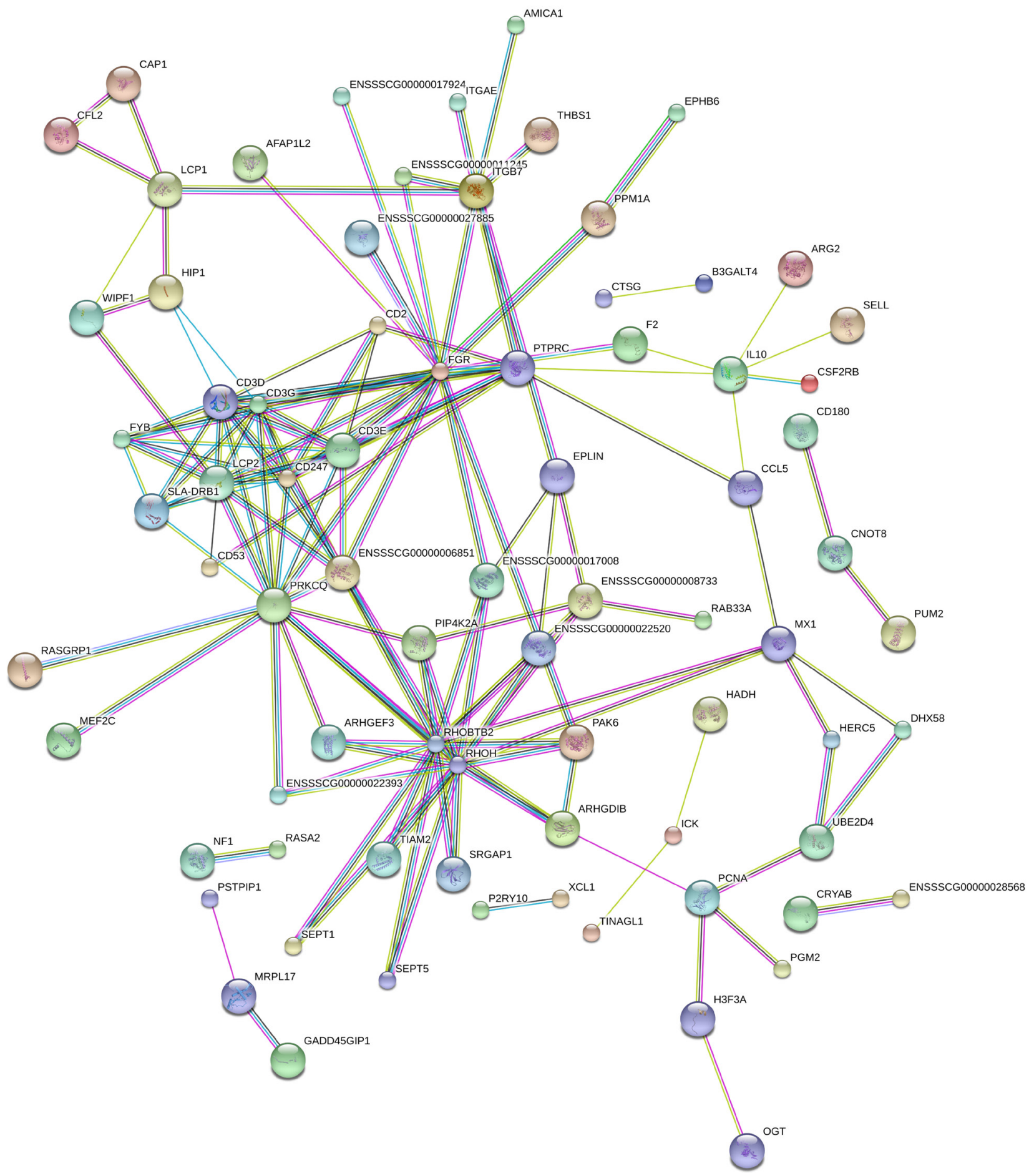

Figure 9: Interaction networks of proteins encoded by DEGs between Con and Res treatment in weaning piglets. Line colors represent different evidentiary sources for each interaction: light blue, from curated databases; purple, experimental; green, gene neighborhood; red, fusion; blue, gene co-occurrence; yellow, text mining; black, co-expression; light purple, protein homology. Disconnected nodes are not shown in the network. 


\section{DISCUSSION}

In our study, weaning-associated diarrhea and inflammation were alleviated by maternal resveratrol supplementation. The intestinal morphology of weaning and post-weaning piglets, including the villus height, villus/crypt ratio and microvillus height, were also improved by maternal resveratrol supplementation. Poudel et al. [16] previously demonstrated that resveratrol can cross the placenta. However, we failed to observe any influence of maternal resveratrol supplementation on intestinal morphology in newborn piglets (data not shown). We did find that IL-1 $\beta$ levels were reduced by maternal dietary resveratrol supplementation (Supplementary Table 9). In this study, newborn piglets were immediately slaughtered after birth without suckling any milk, which indicated that the changes in IL- $1 \beta$ levels may have occurred in utero, and that maternal resveratrol may have a more significant effect on the intestinal function of offspring during lactation. Our results demonstrate that maternal resveratrol is beneficial for the intestinal health of offspring and contributes to the alleviation of weaning-associated diarrhea and inflammation. Weaning is a critical factor related to potential changes in the immune system, intestinal barrier function and absorption, and the endocrine system for infants and young pigs [30]. Unlike in humans, the weaning process for pigs involves an abrupt dietary shift from sow milk to a completely feed-based diet, which consequentially inhibits the intestinal architecture and function, resulting in gutassociated disorders, such as inflammation and diarrhea [27]. Resveratrol exerts strong anti-inflammatory effects in many disease models. Liu et al. [13] demonstrated that resveratrol prevented the damage of villus structures in intestine and pro-inflammatory gene expression induced by heat stress. In addition, dietary resveratrol protected the colonic mucosal architecture, reduced body weight loss, diminished the induced anemia, and reduced systemic inflammation markers, colonic mucosa prostaglandin E2, cycloxygenase-2, prostaglandin E synthase and nitric oxide levels in a colitis model [24]. To our knowledge, our study is the first to demonstrate that maternal resveratrol alleviates diarrhea and inflammation and improves morphology in offspring.

The gastrointestinal microbiota of newborn animals has a profound influence on host health through the regulation of intestinal nutritional metabolism, immune system maturation, and establishment of the gut barrier [22]. Aberrations in the structure and function of gut microbiota, termed microbial dysbiosis, are

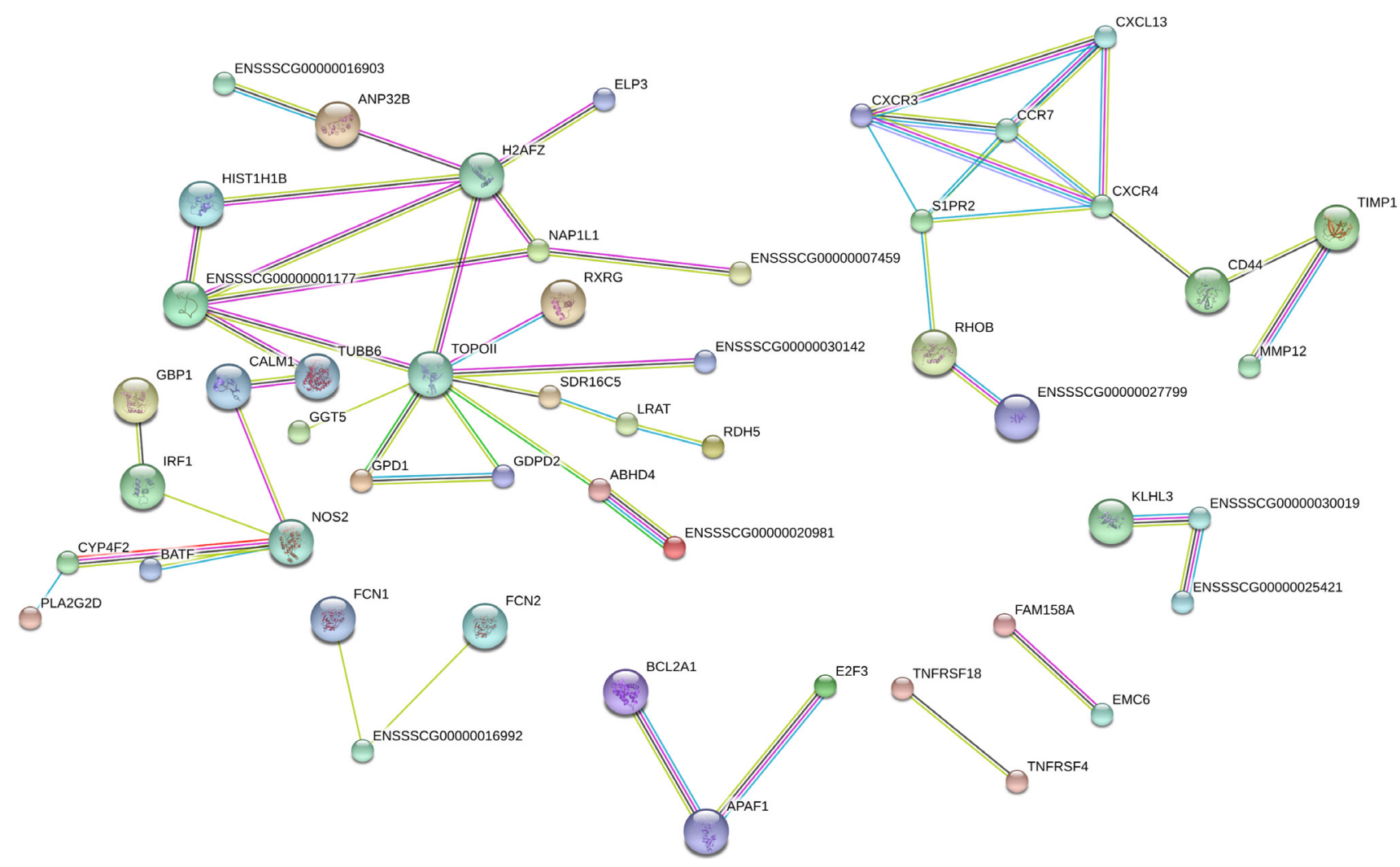

Figure 10: Interaction networks of proteins encoded by DEGs between Con and Res treatment in post-weaning piglets. Line colors represent different evidentiary sources for each interaction: light blue, from curated databases; purple, experimental; green, gene neighborhood; red, fusion; blue, gene co-occurrence; yellow, text mining; black, co-expression; light purple, protein homology. Disconnected nodes are not shown in the network. 
important factors that impact several bowel diseases and inflammatory intestinal disorders [31]. In the present study, we used $16 \mathrm{~S}$ rDNA sequencing to analyze the fecal microbiota in weaning and post-weaning piglets. Firmicutes and Bacteroidetes were predominant in both weaning and post-weaning piglets at the phylum level, which is in agreement with another study [32]. It is believed that intestinal microbiota changes over time from birth to adulthood in response to changes in diet, environmental stress and diseases. In our study, the phylum of Firmicutes was increased and the phyla of Proteobacteria, Cyanobacteria, Verrucomicrobial and Lentisphaerae were reduced by weaning. At the genus level, several genera were increased by the weaning process, such as Ruminococcus, Clostridium IV, Roseburia, Coprococcus, and Peptococcus, among others, along with several decreased genera, including Fusobacterium, Bacteroides, Campylobacter and others. During the weaning process, piglets are switched from breast milk to solid feed, inducing stress and unfavorable changes to the intestinal mucosa and gut physiology that may contribute to changes in the intestinal microbiota [22]. Moreover, the weaning process results in malabsorption of nutrients, immune activation and inflammation, contributing to changes in the intestinal microbiota $[33,34]$.

At the phylum level, we failed to observe any effects of maternal resveratrol on the intestinal microbiota; however, several changes were observed at the genus level. Lachnobacterium in weaning piglets was increased by maternal resveratrol. In 3- to 6- month-old infant, increased Lachnobacterium was linked to vitamin D levels in cord blood [35]. Thus, we speculate that vitamin $\mathrm{D}$ metabolism in piglets may be increased by dietary resveratrol. Indeed, vitamin D has been reported to stimulate the production of pattern recognition receptors, anti-microbial peptides, and cytokines, which are critical for innate immune responses [36, 37]. In our study, the level of Flavonifractor was increased by maternal resveratrol in both weaning and post-weaning piglets. Resveratrol is a phenolic compound found in grapes, red wine and polygonum cuspidatum. Flavonifractor has been demonstrated to convert polyphenols and contribute to butyrate production. In our study, we measured the levels of resveratrol in milk and confirmed that resveratrol could be transported by milk $(0.15 \mu \mathrm{g} / \mathrm{mL})$. Thus, the increased Flavonifractor level may be associated with the resveratrol transported in milk. Moreover, the increasing Flavonifractor level may result in increased butyrate production. The butyrate-producing genera Odoribacter and Oscillibacter in weaning piglets were also increased by maternal resveratrol supplementation [38]. Butyrate, an important energy source for intestinal epithelial cells, has been shown to stimulate growth of small intestinal epithelium and maintain intestinal homeostasis. The effects of butyrate on oxidative stress, inflammation [39], and intestinal barrier function [40] have been described, particularly its activities in promoting gut health both in vitro [41] and in vivo [42]. In addition, previous studies reported that butyrate decreased diarrhea incidence in post-weaning piglets [42] and congenital chloride diarrhea children [43]. In the present study, these increased butyrate-producing bacteria may have been associated with the beneficial effects of maternal resveratrol on the fecal score, intestinal morphology and inflammation.

To further study the mechanism responsible for these effects, we performed a RNA-seq analysis on the jejunums of weaning and post-weaning piglets. We found 189 DEGs, including 70 up-regulated genes and 119 down-regulated genes in weaning piglets. Furthermore, we found 139 DEGs, including 72 up-regulated genes and 67 down-regulated genes, in post-weaning piglets. To validate the DEGs identified by RNA-seq analysis, we confirmed the expression levels of 8 genes in both weaning and postweaning piglets by qRT-PCR. Comparison of the results obtained using the two methods revealed similar trends, confirming their validity.

GO analyses showed that the DEGs of weaning piglets were mostly involved in immune function, including the immune response, inflammatory response, cell migration, cell adhesion, cell surface receptor signaling pathway and innate immune response. After birth, piglets experience a radical change in diet and environment. With an immature immune system, they have to adapt to new conditions and overcome a deficiency in protective elements derived from maternal milk. Genes related to immune cells and inflammation were significantly decreased, including AFAP1L2 (involved in regulation of interleukin-6 production), CCL5 (involved in positive regulation of T cell proliferation), LTF and RASGRP1 (involved in regulation of tumor necrosis factor production) and THBS1 (involved in negative regulation of IL-12 production), which indicated that the inflammatory response was reduced by maternal resveratrol. In addition, KEGG pathway analysis showed that the MAPK and Ras signaling pathways were significantly enriched in weaning piglets. In the MAPK signaling pathway 6 genes were down-regulated, not including HSPB1. The MAPK signaling pathway, which mediates fundamental biological processes and cellular responses to external stress signals, was reduced by maternal resveratrol supplementation. The use of MAPK inhibitors may therefore represent an attractive strategy, as they are capable of reducing both the synthesis of proinflammatory cytokines and their signaling. Resveratrol reportedly exerts anti-inflammatory and anti-apoptosis effects via suppressing MAPK signaling pathway [44, 45]. In addition, the Ras signaling pathway was reduced by maternal resveratrol treatment. The Ras/MAPK pathway is essential for regulation of the cell cycle, differentiation, growth and cell senescence, all of which are critical for normal development [46]. Furthermore, 
modulation of Ras/MAPK signaling has been reported to induce innate immune responses [47].

In weaning piglets, genes involved in cell differentiation and regulation of cell proliferation were down-regulated by maternal resveratrol treatment. Genes related to cell apoptosis, including HIP1 (involved in apoptotic signaling pathway), CD3G (involved in regulation of lymphocyte apoptotic process) and THBS1 (involved in positive regulation of extrinsic apoptotic signaling pathway), were also decreased by maternal resveratrol. Furthermore, genes involved in immune cell differentiation, such as MEF2C, TAL1and TESC (involved in megakaryocyte differentiation), CD3D (involved in T cell differentiation) and RASAL3 (involved in positive regulation of $\mathrm{NK} \mathrm{T}$ cell proliferation), were down-regulated by maternal resveratrol supplementation. Moreover, KEGG pathways analysis revealed that genes enriched in the $\mathrm{T}$ cell receptor signaling pathway, primary immunodeficiency, intestinal immune network for IgA production and cell adhesion molecules were all downregulated by maternal resveratrol treatment in weaning piglets. The delicate balance of intestinal immunity is important for animal health and growth. However, in the present study, we observed that intestinal morphology and inflammation were improved by maternal resveratrol supplementation, which indicated that maternal resveratrol was beneficial for the intestinal health of offspring. In the guts of young animals and children, harmful antigens, such as pathogenic E. coli and other pathogenic bacterial infections, easily induces intestinal immunity activation and enhance inflammation because their immune systems are immature [48]. Previous studies have demonstrated that resveratrol effectively inhibits mitogen/antigeninduced lymphocyte proliferation, $\mathrm{T}$ cell-mediated responses and development of cell-mediated cytotoxicity $[49,50]$. Similarly, previous studies have demonstrated that dietary supplements, such as spray-dried plasma, decrease immune system activation and modify the morphology of the small and large intestines in weaned piglets [51]. Accordingly, we speculate that maternal resveratrol supplementation may inhibit activation of the intestinal immune system and exert beneficial effects on intestinal health in weaning piglets.

In post-weaning piglets, the most significantly enriched GO terms included the oxidation-reduction process, which belongs to biological process, and oxidoreductase activity, which belongs to molecular function. GZMA, involved in negative regulation of oxidoreductase activity, was down-regulated, whereas CYBRD, ND2, OLFML3, RDH5 and SDR16C5, which are involved in oxidation-reduction processes, were up-regulated by maternal resveratrol supplementation, indicating that the oxidative stress in post-weaning piglets was alleviated by maternal resveratrol supplementation. In piglets, Wang et al. [52] demonstrated that early weaning resulted in increased expression of genes related to oxidative stress in the gut by microarray. Yin et al. [30] reported that early weaning stress caused lipid, protein, and DNA oxidative injuries and suppressed NF$\mathrm{B}-\mathrm{p} 65$ and Nrf2 signaling. Accumulating evidences suggest that resveratrol exerts a strong inhibitory effect on the production of reactive oxygen species and free radical scavenging properties in many experimental systems [53-55]. Additional, studies have shown that resveratrol supplementation during pregnancy reduces oxidative stress and apoptosis in diabetic dams embryos and relieves maternal, placental and offspring oxidative stress in a maternal protein restriction model [18, 19]. Moreover, studies have demonstrated that weaning induces inflammatory and pro-inflammatory cytokine production, including TNF- $\alpha$, IL- 6 and INF- $\gamma$, in the gut [28]. Bomba et al. [24] used gene microarray technology to highlight several response mechanisms to weaning stress in the guts of piglets and demonstrated that several important pathways related to immune and inflammatory responses are activated during weaning. In the present study, GO analysis identified genes involved in inflammatory response and response to lipopolysaccharide were down-regulated. KEEG pathway analysis showed that the cytokine-cytokine receptor interaction pathway was significantly reduced, which indicated that the inflammatory state in post-weaning piglets was alleviated by maternal resveratrol supplementation. Chemokine function is critical for all immune cell movements, ranging from the migration required for immune cell development and homeostasis to that required for the generation of primary and amnestic cellular and humoral immune responses [56]. Chemokine are produced in the tissues in response to inflammatory cytokines that are released by local immune cells [56]. In our study, chemokine receptors, including CXCL13, CXCR3, CXCR4 and CCR7, were down-regulated by maternal resveratrol supplementation. CXCL13, also known as B cell-attracting chemokine 1, targets naive $\mathrm{B}$ cells and $\mathrm{CD} 4^{+} \mathrm{T}$ cells during inflammation [57]. CXCR3 is expressed under homeostasis conditions but is up-regulated during inflammation stage. CXCR4 shows abundant apical and basolateral expression on the crypts in both homeostatic and inflammatory conditions [57]. The chemokine receptor CCR7 plays a critical role in the organization of secondary lymphoid tissues, including Payer's patches in the gut. Previous studies have shown that CCR7 coordinates primary immune response by establishing functional microenvironments in secondary lymphoid organs [58]. Moreover, IL-10 receptor subunit alpha (IL10RA) was down-regulated by maternal resveratrol. The anti-inflammatory and pro-inflammatory effects of IL-10 have been described and are dependent on the tissue type and pathological conditions. IL-10 reportedly increases serotonin transporter activity and expression mediated by the IL-10 receptor and is triggered by the PI3K intracellular pathway in intestinal epithelial cells [59]. High endogenous serotonin levels have also 
been reported to be involved in several inflammatory and diarrheal conditions [60]. Thus, the observed IL10RA reduction may be related to the alleviation of diarrhea by maternal resveratrol treatment. TNFRSF4, a receptor for $\mathrm{TNF}-\alpha$, which activates the transcription factor $\mathrm{NF}-\kappa \mathrm{B}$, and regulates inflammation, was also down-regulated, which further revealed that the inflammatory response may be reduced by maternal resveratrol supplementation. GO analysis showed that genes involved in the apoptotic process were also reduced, which indicated that cell apoptosis in post-weaning piglets was reduced by maternal resveratrol.

Interestingly, KEGG pathways analysis of DEGs in post-weaning piglets showed that most were enriched in nutritional metabolism and absorption, including retinol metabolism, arachidonic acid metabolism, glycine, serine and threonine metabolism and vitamin digestion and absorption, which further suggested that the nutritional metabolism and absorption in the intestines of piglets was increased by maternal resveratrol supplementation. These effects may be due to the improvement in intestinal morphology, and the subsequent nutritional metabolism and absorption effects are affected by maternal resveratrol supplementation.

In this study, we demonstrated that maternal resveratrol alleviated weaning-associated diarrhea and intestinal inflammation and improved intestinal morphology in porcine offspring, potentially due to the modulation of intestinal gene expression and microbiota. $16 \mathrm{~S}$ rDNA sequencing analysis showed that maternal resveratrol supplementation increased the proportions of butyrate-producing bacteria in the feces of weaning piglets. Intestinal RNA-seq analysis showed that maternal resveratrol supplementation down-regulated the expression of genes involved in the $\mathrm{T}$ cell receptor, MAPK and Ras signaling pathways in weaning piglets. In addition, maternal resveratrol supplementation downregulated the expression of genes involved in the cytokinecytokine receptor interaction pathway in post-weaning piglets.

\section{MATERIALS AND METHODS}

\section{Animal husbandry}

The protocols used in this experiment were approved by the Northeast Agricultural University Institutional Animal Care and Use Committee. All of the animal experimental procedures were approved by the Ethical and Animal Welfare Committee of Heilongjiang Province, China.

Forty sows (average parity 4.4) were bred with semen from a pool of Landrace boars. Sows were segregated into 2 dietary treatments 20 days after breeding as follows: 1) control sows were fed a corn-soybean meal basal diet (Con treatment, $n=20$ ), and 2) treatment sows were fed a basal diet supplemented with $300 \mathrm{mg} / \mathrm{kg}$ resveratrol for 20 days after breeding through gestation and lactation (Res treatment, $n=20)$ ). Resveratrol (98\% purity) extracted from Polygonum cuspidatum was purchased from Jing Zhu Biotechnology Co. Ltd (Nanjing, Shanxi province, China). The sows were kept in single crates $(0.6 \times 2.0 \mathrm{~m})$ from insemination to day 110 of gestation. On day 110 of gestation, sows were transported to the farrowing facility, where they were placed in individual farrowing crates $(2.4 \times 2.4 \mathrm{~m})$. Farrowing crates were equipped with 2 stainless-steel water nipples, 1 for sows and 1 for piglets, and stainless-steel feeders, in which sows were provided diets. Each crate had steel mesh floors with a heat lamp for newborn piglets. The crates were mounted over a solid concrete floor, and manure was removed manually each day. The farrowing room temperature was maintained at approximately 18 to $20^{\circ} \mathrm{C}$. Experimental diets (Supplementary Table 10) were formulated according to the nutrient requirements of swine [61]. From day 20 until day 90 of gestation, all sows were daily fed $2.5 \mathrm{~kg}$ gestation diets. Starting at day 91 of gestation, all sows were daily fed $3.5 \mathrm{~kg}$ gestation diets. The amounts of parturition feed provided to each sow at days 112, 113 and 114 of gestation were 2.0, 1.5 and $1.0 \mathrm{~kg}$, respectively. On the day of farrowing, sows were offered $2.0 \mathrm{~kg}$ of the lactation diet. Thereafter, this amount was increased by $1.0 \mathrm{~kg}$ daily until it reached ad libitum feeding. The piglets were cross-fostered within dietary treatment groups within $24 \mathrm{~h}$ of birth to standardize the litter size to range between 9 and 12 piglets, and this was maintained throughout the lactation period. At day 7, piglets received an iron injection, and males were castrated. The piglets were weaned on day 21 of lactation. On the weaning day, the sows were removed from the piglets, while the piglets remained in the nursing pens for a week until day 28 to avoid the stress caused by a change in environment. The birth weight and the body weight of the piglets on day 28 were recorded to calculate the body weight gain. Fecal scores were recorded daily from each pen. Fecal consistency scoring was performed by one trained person with no prior knowledge of the dietary treatment.

\section{Sample and data collection}

Six litters per treatment were randomly selected for sample collection at both weaning (day 21) and 7 days post-weaning (day 28). Weaning piglets ( $n=6$ per treatment, 1 piglet per litter) and 7-day post-weaning piglets ( $n=6$ per treatment, 1 piglet per litter), regardless of sex, were randomly selected and slaughtered by an intra-arterial injection of pentobarbital $(200 \mathrm{mg} / \mathrm{kg}$ ) after general anesthesia. Then, fresh feces of weaning piglets and 7-day post-weaning piglets were collected from each pen and stored immediately at $-80^{\circ} \mathrm{C}$ for microbiota analysis. After slaughter, jejunal sections 
were immediately excised and rinsed in normal saline. Afterwards, the samples were quickly collected for further analysis.

\section{Intestinal morphology}

Jejunum sample were fixed in 4\% paraformaldehyde. The segments of jejunum samples were embedded in paraffin wax and then sectioned, followed by staining with hematoxylin and eosin. Each slide was divided into three single segments, and the morphology of jejunum was analyzed using an optical microscope (Nikon Eclipse E400). Thirty intact and well-oriented crypt-villus units per sample were selected randomly and measured. Crypt depth and villus length were measured using Image-Pro Plus software (Version 6.0, Media Cybernetics, Silver Spring, MD, USA).

\section{Scanning electron microscopy (SEM)}

Jejunum samples were fixed with $2.5 \%$ glutaraldehyde, followed with $1 \%$ osmium tetroxide for $2 \mathrm{~h}$ at $4^{\circ} \mathrm{C}$. The samples were dehydrated with an increasing gradient of ethanol for $20 \mathrm{~min}$ at each step and then transferred into a mixture $(\mathrm{v}: \mathrm{v}=1: 1)$ of alcohol and tert-butanol for $30 \mathrm{~min}$, followed by andtert-butanol for $1 \mathrm{~h}$. Then, the samples were dehydrated in a Hitachi Model HCP-2 critical point dryer with liquid $\mathrm{CO}_{2}$. The dried samples were coated with gold-palladium prior to observation using a digital SEM (Hitachi S-4800, Japan).

\section{Transmission electron microscopy (TEM)}

TEM was used to observe microvilli in the jejunum. Jejunum samples were fixed with $2.5 \%$ glutaraldehyde at $4^{\circ} \mathrm{C}$. The samples were washed twice with PBS, post-fixed with $2 \%$ osmium tetroxide for $70 \mathrm{~min}$, and washed twice with PBS. Then, the jejunum tissue was dehydrated and transferred into pure acetone for $20 \mathrm{~min}$. The specimens were placed into a mixture of acetone and epoxy resin mixture $(1: 1$ for $1 \mathrm{~h}$ and $1: 3$ for $3 \mathrm{~h}$ ) and then transferred into pure epoxy resin overnight at a constant temperature. The tissue sections were placed in capsules containing embedding medium and heated at $70^{\circ} \mathrm{C}$ for $9 \mathrm{~h}$. Finally, the specimens were sectioned with an ultramicrotome, stained with uranyl acetate and lead citrate, and observed using a Hitachi H-7650 TEM.

\section{Assessment of pro-inflammatory cytokines}

Jejunum tissue homogenates $(10 \%)$ were analyzed to assess pro-inflammatory cytokine levels. Commercial ELISA kits were used to detect IL-1 $\beta$, IL-6, IL-8, and TNF- $\alpha$ levels (Cusabio, Wuhan, China).

\section{S rDNA sequencing: DNA extractions, PCR amplification and sequencing}

DNA from fecal samples was extracted using the Stool DNA Kit (Omega, Inc., USA) according to the manufacturer's instructions. Total DNA was eluted in $50 \mu \mathrm{L}$ of elution buffer by a modification of the procedure described by the manufacturer (QIAGEN) and stored at $-80^{\circ} \mathrm{C}$ until measurement via PCR amplification. The V3$\mathrm{V} 4$ region of the bacterial $16 \mathrm{~S}$ rRNA was amplified with the primers (F: 5'-ACTCCTACGGGAGGCAGCAG-3'; R: 5'-GGACTACHVGGGTWTCTAAT-3'). PCR analysis was performed in triplicate using $25 \mathrm{~mL}$ reactions containing $25 \mathrm{ng}$ of template DNA, $12.5 \mu \mathrm{L}$ PCR Premix, $2.5 \mu \mathrm{L}$ of each primer, and PCR-grade water to equalize the final volumes. PCR reactions were performed under the following conditions: initial denaturation at $98^{\circ} \mathrm{C}$ for $30 \mathrm{~s} ; 35$ cycles of denaturation at $98^{\circ} \mathrm{C}$ for $10 \mathrm{~s}$, annealing at $54^{\circ} \mathrm{C}$ for $30 \mathrm{~s}$, and extension at $72^{\circ} \mathrm{C}$ for $45 \mathrm{~s}$; and a final extension at $72^{\circ} \mathrm{C}$ for $10 \mathrm{~min}$. The PCR products were confirmed with $2 \%$ agarose gel electrophoresis. The PCR products were purified with AMPure XT beads (Beckman Coulter Genomics, Danvers, MA, USA) and quantified by Qubit (Invitrogen, CA, USA). The amplicon pools were prepared for sequencing, and the size and quantity of the amplicon library were assessed on an Agilent 2100 bioanalyzer (Agilent, USA) and with the Library Quantification Kit for Illumina (Kapa Biosciences, Woburn, MA, USA). A PhiX control library (Illumina) was combined with the amplicon library (expected at $30 \%$ ). The libraries were sequenced via 300PE MiSeq runs, and one library was sequenced with both protocols using standard Illumina sequencing primers, eliminating the need for a third (or fourth) index read.

\section{S rDNA sequencing: data analysis}

Samples were sequenced on an Illumina MiSeq platform according to the manufacturer's recommendations, provided by LC-Bio. Paired-end reads were assigned to samples based on their unique barcodes and truncated by removing the barcode and primer sequence. Paired-end reads were merged using FLASH. Quality filtering on the raw tags was performed under specific filtering conditions to obtain high-quality clean tags according to FastQC (V 0.10.1). Chimeric sequences were filtered using Verseach software. Sequences were assigned to OTUs at $97 \%$ similarity. Representative sequences were chosen for each OTU, and taxonomic data were then assigned to each representative sequence using the RDP classifier (Ribosomal Database Project). To estimate alpha diversity, we calculated the Chao 1 (species richness estimator), Shannon and Simpson indices (diversity indices) [62]. 


\section{RNA-Seq: RNA extraction and sequencing}

Total RNA was extracted using Trizol reagent (Invitrogen, CA, USA) according to the manufacturer's procedure. The total RNA quantity and purity were evaluated with an Agilent Technologies 2100 bioanalyzer with RNA integrity number (RIN) values greater than 7.5. A cDNA library was constructed from the pooled RNA and sequenced on the Illumina 4000 sequence platform. Using the Illumina paired-end RNA-seq approach, we sequenced the transcriptome, generating a total of 20 million paired-end reads of $150 \mathrm{bp}$ length. Prior to assembly, the low-quality reads $(1$, reads containing sequencing adaptors; 2 , reads containing sequencing primers; and 3, nucleotides with q quality scores lower than 20) were removed. The RNA-seq data sets have been deposited in Gene Expression Omnibus (GEO) database under accession number GSE107474.

\section{RNA-Seq: mapping and identification of differentially expressed mRNAs}

We aligned sample reads to the Ensembl (http:// www.ensembl.org/) pig reference genome using TopHat, which initially removes a portion of the reads based on the quality information accompanying each read and then maps the reads to the reference genome. TopHat allows multiple alignments per read (up to 20 by default) and a maximum of two mismatches when mapping the reads to the reference. TopHat builds a database of potential splice junctions and confirms these by comparing the previously unmapped reads against the database of putative junctions. The mapped reads of each sample were assembled using StringTie. Then, all transcriptomes from the samples were merged to reconstruct a comprehensive transcriptome using Perl scripts. After the final transcriptome was generated, StringTie and Ball gown were used to estimate the expression levels of all genes. StringTie was used to analyze gene expression levels by calculating the FPKM. The differentially expressed genes were selected with $\log 2$ (fold-change) $>1$ or $\log 2$ (fold-change) $<-1$ and with statistical significance $(P<0.05)$ by R package-Ball gown.

\section{RNA-Seq: GO term enrichment and KEGG Pathway analyses}

GO enrichment analysis of the DEGs was performed with the GOseq R package. KOBAS software was used to test the statistical enrichment of DEGs in the KEGG pathways analysis. $P<0.05$ was considered significant for $\mathrm{GO}$ and KEGG enrichment.

\section{Quantitative real-time PCR (qRT-PCR) validation}

Total RNA from each sample was converted into cDNA using the Prime Script RT reagent Kit (TaKaRa Bio
Catalog) and used for qRT-PCR. The primer sequences are shown in Supplementary Table 11. qRT-PCR was performed using the SYBR Green I Kit (TaKaRa Bio Catalog). For analyses on an ABI PRISM 7500 SDS thermal cycler, PCR reactions were performed with 2.0 $\mathrm{mL}$ of first-strand cDNA and $0.4 \mathrm{~mL}$ of forward and reverse primers in a final volume of $20 \mathrm{~mL}$. Samples were centrifuged briefly and run on the PCR machine using the default fast program $\left(1\right.$ cycle at $95^{\circ} \mathrm{C}$ for $30 \mathrm{~s}, 40$ cycles of $95^{\circ} \mathrm{C}$ for $5 \mathrm{~s}$ and $60^{\circ} \mathrm{C}$ for $34 \mathrm{~s}$ ). All of the PCR reactions were performed in triplicate. The relative gene expression levels were determined using the $2^{-\Delta \Delta \mathrm{Ct}}$ method [63].

\section{Interaction networks between proteins encoded by DEGs}

The known and predicted interaction networks between proteins encoded by DEGs were obtained using the STRING database. STRING is a web-based tool that reflects and predicts the interrelationship between proteins, including direct physical interactions and also highlights proteins that are common between metabolic pathways or biological processes.

\section{Statistical analysis}

All data analyses were performed with SPSS 18.0 software (IBM-SPSS Inc., Chicago, Illinois, USA). The data of fecal scores, growth performance and qRT-PCR were analyzed by one-way analysis of variance (ANOVA) and multiple comparisons with the Tukey's test in SPSS. The data of intestinal morphology, microbiota and proinflammatory were analyzed by two-way ANOVA to establish the effect of maternal dietary resveratrol and weaning or any interaction between these two factors. Differences were considered significant at $P<0.05$ and $\mathrm{P}$ values between 0.05 and 0.10 were deemed trends.

\section{CONFLICTS OF INTEREST}

The authors declare that they have no competing interests.

\section{FUNDING}

This work was supported by the China Agriculture Research System (CARS-35), the National Key Research and Development Plan of China (2016YFD0501207), and the National Basic Research Program (2012CB124703).

\section{REFERENCES}

1. Lalles JP, Boudry G, Favier C, Le Floc'h N, Lurona I, Montagne L, Oswald IP, Pie S, Piel C, Seve B. Gut function and dysfunction in young pigs: physiology. Anim Res. 2004; 53:301-316. 
2. Han M, Song P, Huang C, Rezaei A, Farrar S, Brown MA, Ma X. Dietary grape seed proanthocyanidins (GSPs) improve weaned intestinal microbiota and mucosal barrier using a piglet model. Oncotarget. 2016; 7:80313-80326. https://doi.org/10.18632/oncotarget.13450.

3. Allen HK, Looft T, Bayles DO, Humphrey S, Levine UY, Alt D, Stanton TB. Antibiotics in feed induce prophages in swine fecal microbiomes. MBio. 2011; 2:2.

4. Zhong HJ, Li H, Liu GM, Wan HF, Mercier Y, Zhang XL, Lin Y, Che LQ, Xu SY, Tang L, Tian G, Chen DW, Wu D, Fang ZF. Increased maternal consumption of methionine as its hydroxyl analog promoted neonatal intestinal growth without compromising maternal energy homeostasis. J Anim Sci Biotechnol. 2016; 7:46.

5. Leonard SG, Sweeney T, Bahar B, Lynch BP, O'Doherty JV. Effect of dietary seaweed extracts and fish oil supplementation in sows on performance, intestinal microflora, intestinal morphology, volatile fatty acid concentrations and immune status of weaned pigs. Br J Nutr. 2011; 105:549-560.

6. Baur JA, Sinclair DA. Therapeutic potential of resveratrol: the in vivo evidence. Nat Rev Drug Discov. 2006; 5:493-506.

7. Liu Y, Wang YL, He SW, Chen MH, Zhang Z, Fu XP, Fu BB, Liao BQ, Lin YH, Qi ZQ, Wang HL. Protective effects of resveratrol against mancozeb induced apoptosis damage in mouse oocytes. Oncotarget. 2017; 8:6233-45. https://doi. org/10.18632/oncotarget.14056.

8. Signorelli P, Ghidoni R. Resveratrol as an anticancer nutrient: molecular basis, open questions and promises. J Nutr Biochem. 2005; 16:449-466.

9. Bonnefont-Rousselot D. Resveratrol and Cardiovascular Diseases. Nutrients. 2016; 8:250.

10. Tellone E, Galtieri A, Russo A, Giardina B, Ficarra S. Resveratrol: A Focus on Several Neurodegenerative Diseases. Oxid Med Cell Longev. 2015; 2015:392169.

11. Etxeberria U, Arias N, Boqué N, Macarulla MT, Portillo MP, Martínez JA, Milagro FI. Reshaping faecal gut microbiota composition by the intake of trans-resveratrol and quercetin in high-fat sucrose diet-fed rats. J Nutr Biochem. 2015; 26:651-60.

12. Yildiz F, Terzi A, Coban S, Celik H, Aksoy N, Bitiren M, Cakir H, Ozdogan MK. Protective effects of resveratrol on small intestines against intestinal ischemia-reperfusion injury in rats. J Gastroenterol Hepatol. 2009; 24:1781-85.

13. Liu L, Fu C, Yan M, Xie H, Li S, Yu Q, He S, He J. Resveratrol modulates intestinal morphology and HSP70/90, NF-kappaB and EGF expression in the jejunal mucosa of black-boned chickens on exposure to circular heat stress. Food Funct. 2016; 7:1329-1338.

14. Sanchez-Fidalgo S, Cardeno A, Villegas I, Talero E, de la Lastra CA. Dietary supplementation of resveratrol attenuates chronic colonic inflammation in mice. Eur $\mathrm{J}$ Pharmacol. 2010; 633:78-84.
15. Franco JG, Dias-Rocha CP, Fernandes TP, Albuquerque Maia L, Lisboa PC, Moura EG, Pazos-Moura CC, Trevenzoli IH. Resveratrol treatment rescues hyperleptinemia and improves hypothalamic leptin signaling programmed by maternal high-fat diet in rats. Eur J Nutr. 2016; 55:601-610.

16. Poudel R, Stanley JL, Rueda-Clausen CF, Andersson IJ, Sibley CP, Davidge ST, Baker PN. Effects of resveratrol in pregnancy using murine models with reduced blood supply to the uterus. PLoS One. 2013; 8:e64401.

17. Yao L, Wan J, Li H, Ding J, Wang Y, Wang X, Li M. Resveratrol relieves gestational diabetes mellitus in mice through activating AMPK. Repro d Biol Endocrinol. 2015; 13:118.

18. Vega CC, Reyes-Castro LA, Rodriguez-Gonzalez GL, Bautista CJ, Vazquez-Martinez M, Larrea F, ChamorroCevallos GA, Nathanielsz PW, Zambrano E. Resveratrol partially prevents oxidative stress and metabolic dysfunction in pregnant rats fed a low protein diet and their offspring. J Physiol-London. 2016; 594:1483-1499.

19. Singh CK, Kumar A, Hitchcock DB, Fan D, Goodwin R, LaVoie HA, Nagarkatti P, DiPette DJ, Singh US. Resveratrol prevents embryonic oxidative stress and apoptosis associated with diabetic embryopathy and improves glucose and lipid profile of diabetic dam. Mol Nutr Food Res. 2011; 55:1186-1196.

20. Flint HJ, Scott KP, Louis P, Duncan SH. The role of the gut microbiota in nutrition and health. Nat Rev Gastroenterol Hepatol. 2012; 9:577-89.

21. Mach N, Berri M, Estelle J, Levenez F, Lemonnier G, Denis C, Leplat JJ, Chevaleyre C, Billon Y, Dore J, RogelGaillard C, Lepage P. Early-life establishment of the swine gut microbiome and impact on host phenotypes. Environ Microbiol Rep. 2015; 7:554-569.

22. Frese SA, Parker K, Calvert CC, Mills DA. Diet shapes the gut microbiome of pigs during nursing and weaning. Microbiome. 2015; 3:28.

23. Qiao Y, Sun J, Xia S, Tang X, Shi Y, Le G. Effects of resveratrol on gut microbiota and fat storage in a mouse model with high-fat-induced obesity. Food Funct. 2014; 5:1241-49.

24. Larrosa M, Yanez-Gascon MJ, Selma MV, GonzalezSarrias A, Toti S, Ceron JJ, Tomas-Barberan F, Dolara P, Espin JC. Effect of a low dose of dietary resveratrol on colon microbiota, inflammation and tissue damage in a DSS-induced colitis rat model. J Agric Food Chem. 2009; 57:2211-2220.

25. Merrifield CA, Lewis M, Claus SP, Beckonert OP, Dumas ME, Duncker S, Kochhar S, Rezzi S, Lindon JC, Bailey M, Holmes E, Nicholson JK. A metabolic system-wide characterisation of the pig: a model for human physiology. Mol Biosyst. 2011; 7:2577-88.

26. Heim G, Sweeney T, O'Shea CJ, Doyle DN, O’Doherty JV. Effect of maternal dietary supplementation of laminarin and 
fucoidan, independently or in combination, on pig growth performance and aspects of intestinal health. Anim Feed Sci Tech. 2015; 204:28-41.

27. Pluske JR. Invited review: Aspects of gastrointestinal tract growth and maturation in the pre- and postweaning period of pigs. J Anim Sci. 2016; 94:399-411.

28. $\mathrm{Hu} \mathrm{CH}$, Xiao K, Luan ZS, Song J. Early weaning increases intestinal permeability, alters expression of cytokine and tight junction proteins, and activates mitogen-activated protein kinases in pigs. J Anim Sci. 2013; 91:1094-1101.

29. Wang Z, Gerstein M, Snyder M. RNA-Seq: a revolutionary tool for transcriptomics. Nat Rev Genet. 2009; 10:57-63.

30. Yin J, Wu MM, Xiao H, Ren WK, Duan JL, Yang G, Li TJ, Yin YL. Development of an antioxidant system after early weaning in piglets. J Anim Sci. 2014; 92:612-619.

31. Yang Q, Huang X, Zhao S, Sun W, Yan Z, Wang P, Li S, Huang W, Zhang S, Liu L, Gun S. Structure and Function of the Fecal Microbiota in Diarrheic Neonatal Piglets. Front Microbiol. 2017; 8:502.

32. Bian G, Ma S, Zhu Z, Su Y, Zoetendal EG, Mackie R, Liu J, Mu C, Huang R, Smidt H, Zhu W. Age, introduction of solid feed and weaning are more important determinants of gut bacterial succession in piglets than breed and nursing mother as revealed by a reciprocal cross-fostering model. Environ Microbiol. 2016; 18:1566-1577.

33. Montagne L, Boudry G, Favier C, Le Huerou-Luron I, Lalles JP, Seve B. Main intestinal markers associated with the changes in gut architecture and function in piglets after weaning. Br J Nutr. 2007; 97:45-57.

34. Merrifield CA, Lewis MC, Claus SP, Pearce JT, Cloarec O, Duncker S, Heinzmann SS, Dumas ME, Kochhar S, Rezzi S, Mercenier A, Nicholson JK, Bailey M, et al. Weaning diet induces sustained metabolic phenotype shift in the pig and influences host response to Bifidobacterium lactis NCC2818. Gut. 2013; 62:842-851.

35. Sordillo JE, Zhou Y, McGeachie MJ, Ziniti J, Lange N, Laranjo N, Savage JR, Carey V, O'Connor G, Sandel M, Strunk R, Bacharier L, Zeiger R, et al. Factors influencing the infant gut microbiome at age 3-6 months: Findings from the ethnically diverse Vitamin D Antenatal Asthma Reduction Trial (VDAART). J Allergy Clin Immun. 2017; 139:482-491.e414.

36. Liu PT, Stenger S, Li H, Wenzel L, Tan BH, Krutzik SR, Ochoa MT, Schauber J, Wu K, Meinken C, Kamen DL, Wagner M, Bals R, et al. Toll-like receptor trigg ering of a vitamin D-mediated human antimicrobial response. Science. 2006; 311:1770-73.

37. Dimitrov V, White JH. Vitamin D signaling in intestinal innate immunity and homeostasis. Mol Cell Endocrinol. 2017; 453:68-78.

38. Gomez-Arango LF, Barrett HL, McIntyre HD, Callaway LK, Morrison M, Dekker Nitert M, and SPRING Trial Group. Increased Systolic and Diastolic Blood Pressure Is
Associated With Altered Gut Microbiota Composition and Butyrate Production in Early Pregnancy. Hypertension. 2016; 68:974-81.

39. Canani RB, Costanzo MD, Leone L, Pedata M, Meli $\mathrm{R}$, Calignano A. Potential beneficial effects of butyrate in intestinal and extraintestinal diseases. World $\mathrm{J}$ Gastroenterol. 2011; 17:1519-1528.

40. Ma X, Fan PX, Li LS, Qiao SY, Zhang GL, Li DF. Butyrate promotes the recovering of intestinal wound healing through its positive effect on the tight junctions. J Anim Sci. 2012; 90:266-268.

41. Dou X, Han J, Song W, Dong N, Xu X, Zhang W, Shan A. Sodium butyrate improves porcine host defense peptide expression and relieves the inflammatory response upon toll-like receptor 2 activation and histone deacetylase inhibition in porcine kidney cells. Oncotarget. 2017; 8:26532-51. https://doi.org/10.18632/oncotarget.15714.

42. Huang C, Song P, Fan P, Hou C, Thacker P, Ma X. Dietary Sodium Butyrate Decreases Postweaning Diarrhea by Modulating Intestinal Permeability and Changing the Bacterial Communities in Weaned Piglets. J Nutr. 2015; 145:2774-2780.

43. Canani RB, Terrin G, Cirillo P, Castaldo G, Salvatore F, Cardillo G, Coruzzo A, Troncone R. Butyrate as an effective treatment of congenital chloride diarrhea. Gastroenterology. 2004; 127:630-634.

44. El-Mowafy AM, White RE. Resveratrol inhibits MAPK activity and nuclear translocation in coronary artery smooth muscle: reversal of endothelin-1 stimulatory effects. FEBS Lett. 1999; 451:63-67.

45. Guo R, Su Y, Liu B, Li S, Zhou S, Xu Y. Resveratrol suppresses oxidised low-density lipoprotein-induced macrophage apoptosis through inhibition of intracellular reactive oxygen species generation, LOX-1, and the p38 MAPK pathway. Cell Physiol Biochem. 2014; 34:603-16.

46. Tidyman WE, Rauen KA. The RASopathies: developmental syndromes of Ras/MAPK pathway dysregulation. Curr Opin Genet Dev. 2009; 19:230-236.

47. Ragab A, Buechling T, Gesellchen V, Spirohn K, Boettcher AL, Boutros M. Drosophila Ras/MAPK signalling regulates innate immune responses in immune and intestinal stem cells. EMBO J. 2011; 30:1123-36.

48. Liu Y, Song M, Che TM, Lee JJ, Bravo D, Maddox CW, Pettigrew JE. Dietary plant extracts modulate gene expression profiles in ileal mucosa of weaned pigs after an Escherichia coli infection. J Anim Sci. 2014; 92:2050-2062.

49. Gao X, Xu YX, Janakiraman N, Chapman RA, Gautam SC. Immunomodulatory activity of resveratrol: suppression of lymphocyte proliferation, development of cell-mediated cytotoxicity, and cytokine production. Biochem Pharmacol. 2001; 62:1299-1308.

50. Gao X, Deeb D, Media J, Divine G, Jiang H, Chapman RA, Gautam SC. Immunomodulatory activity of resveratrol: 
discrepant in vitro and in vivo immunological effects. Biochem Pharmacol. 2003; 66:2427-2435.

51. Nofrarías M, Manzanilla EG, Pujols J, Gibert X, Majó N, Segalés J, Gasa J. Spray-dried porcine plasma affects intestinal morphology and immune cell subsets of weaned pigs . Livest Sci. 2007; 108:299-302.

52. Wang J, Chen L, Li P, Li X, Zhou H, Wang F, Li D, Yin Y, $\mathrm{Wu} \mathrm{G}$. Gene expression is altered in piglet small intestine by weaning and dietary glutamine supplementation. J Nutr. 2008; 138:1025-32.

53. de la Lastra CA, Villegas I. Resveratrol as an antioxidant and pro-oxidant agent: mechanisms and clinical implications. Biochem Soc Trans. 2007; 35:1156-60.

54. Mahal HS, Mukherjee T. Scavenging of reactive oxygen radicals by resveratrol: antioxidant effect. Res Chem Intermed. 2006; 32:59-71.

55. Fremont L. Biological effects of resveratrol. Life Sci. 2000; 66:663-673.

56. Griffith JW, Sokol CL, Luster AD. Chemokines and chemokine receptors: positioning cells for host defense and immunity. Annu Rev Immunol. 2014; 32:659-702.

57. Zimmerman NP, Vongsa RA, Wendt MK, Dwinell MB. Chemokines and chemokine receptors in mucosal homeostasis at the intestinal epithelial barrier in inflammatory bowel disease. Inflamm Bowel Dis. 2008; 14:1000-11.
58. Förster R, Schubel A, Breitfeld D, Kremmer E, RennerMüller I, Wolf E, Lipp M. CCR7 coordinates the primary immune response by establishing functional microenvironments in secondary lymphoid organs. Cell. 1999; 99:23-33.

59. Latorre E, Mendoza C, Matheus N, Castro M, Grasa L, Mesonero JE, Alcalde AI. IL-10 modulates serotonin transporter activity and molecular expression in intestinal epithelial cells. Cytokine. 2013; 61:778-784.

60. Spiller R. Recent advances in understanding the role of serotonin in gastrointestinal motility in functional bowel disorders: alterations in 5-HT signalling and metabolism in human disease. Neurogastroenterol Motil. 2007; 19:25-31.

61. NRC. Nutrient requirements of swine. 11th rev. ed. Washington (DC): National Academy Press. 2012.

62. Schloss PD, Westcott SL, Ryabin T, Hall JR, Hartmann M, Hollister EB, Lesniewski RA, Oakley BB, Parks DH, Robinson CJ, Sahl JW, Stres B, Thallinger GG, et al. Introducing mothur: open-source, platform-independent, community-supported software for describing and comparing microbial communities. Appl Environ Microbiol. 2009; 75:7537-41.

63. Livak KJ, Schmittgen TD. Analysis of relative gene expression data using real-time quantitative PCR and the 2- $\Delta \Delta$ CT method. Methods. 2001; 25:402-408. 